\title{
Canonical TGF $\beta$ signaling induces collective invasion in colorectal carcinogenesis through a Snail1- and Zeb1-independent partial EMT
}

Marion Flum ${ }^{1,2,3}$, Severin Dicks ${ }^{3,4}$, Monika Schrempp ${ }^{1}$, Alexander Nyström ${ }^{5}$, Melanie Boerries $^{4,6,7,8}$, and Andreas Hecht ${ }^{1,3,9,{ }^{*}}$

${ }^{1}$ Institute of Molecular Medicine and Cell Research, Faculty of Medicine, University of Freiburg, 79104 Freiburg, Germany

${ }^{2}$ Spemann Graduate School of Biology and Medicine (SGBM), University of Freiburg, 79104 Freiburg, Germany

${ }^{3}$ Faculty of Biology, University of Freiburg, 79104 Freiburg, Germany

${ }^{4}$ Institute of Medical Bioinformatics and Systems Medicine, Medical Center - University of Freiburg, Faculty of Medicine, University of Freiburg, 79110 Freiburg, Germany

${ }^{5}$ Department of Dermatology, Medical Center - University of Freiburg, Faculty of Medicine, University of Freiburg, 79104 Freiburg, Germany.

${ }^{6}$ German Cancer Consortium (DKTK), Freiburg, University of Freiburg, 79104 Freiburg, Germany

${ }^{7}$ German Cancer Research Center (DKFZ), 69120 Heidelberg, Germany

${ }^{8}$ Comprehensive Cancer Center Freiburg (CCCF), Medical Center - University of Freiburg, Faculty of Medicine, University of Freiburg, 79106 Freiburg, Germany

${ }^{9}$ BIOSS Centre for Biological Signalling Studies, University of Freiburg, 79104 Freiburg, Germany

${ }^{\#}$ Corresponding author (ORCID iD 0000-0003-2262-2575):

Andreas Hecht

Institute of Molecular Medicine and Cell Research

Albert-Ludwigs-University Freiburg

Stefan-Meier-Str. 17 


\section{D-79104 Freiburg, Germany}

Phone: +49-761-203 9608

Fax: +49-761-203 9602

E-mail: andreas.hecht@mol-med.uni-freiburg.de 


\section{Abstract}

Local invasion is the initial step towards metastasis, the main cause of cancer mortality. In human colorectal cancer (CRC), malignant cells predominantly invade as cohesive collectives, and may undergo partial epithelial-mesenchymal transition (pEMT) at the invasive front. How this particular mode of stromal infiltration is generated is unknown. Here we investigated the impact of oncogenic transformation and the microenvironment on tumor cell invasion using genetically engineered organoids as CRC models. We found that inactivation of the Apc tumor suppressor combined with expression of oncogenic Kras ${ }^{G 12 D}$ and dominant negative $\operatorname{Trp5} 3^{R 172 H}$ did not cell-autonomously induce invasion in vitro. However, oncogenic transformation primed organoids for activation of a collective invasion program upon exposure to the prototypical microenvironmental factor TGF $\beta 1$. Execution of this program co-depended on a permissive extracellular matrix which was further actively remodeled by invading organoids. Although organoids shed some epithelial properties particularly at the invasive edge, TGF $\beta 1$-stimulated organoids largely maintained epithelial gene expression while additionally implementing a mesenchymal transcription pattern, resulting in a pEMT phenotype that did not progress to a fully mesenchymal state. Induction of this stable pEMT required canonical, Smad4-mediated TGF $\beta$ signaling, whereas the EMT master regulators Snail1 and Zeb1 were dispensable. Gene expression profiling provided further evidence for $\mathrm{pEMT}$ of TGF $\beta 1$-treated organoids and showed that their transcriptomes resemble those of human poor prognosis CMS4 cancers which likewise exhibit pEMT features. We propose that collective invasion in colorectal carcinogenesis is triggered by microenvironmental stimuli through activation of a novel, transcription-mediated form of nonprogressive pEMT independently of classical EMT regulators. 


\section{Introduction}

Metastasis - accountable for the overwhelming majority of cancer-related deaths in solid cancers - requires that tumor cells successfully pass through a series of events summarily termed invasion-metastasis cascade (1). Tumor cells can accomplish the initial step of this cascade by shedding cell-cell contacts and infiltrate adjacent stromal tissue as individual cells, employing amoeboid or mesenchymal modes of migration (2). However, most human cancers display a collective mode of invasion where tumor cells maintain intercellular interactions and migrate in cohesive groups (2). Nonetheless, cells at the invasive front of such a collective may also display mesenchymal features $(2,3)$. Unfortunately, knowledge about the prerequisites and molecular determinants which promote a specific mode of invasion is limited.

Epithelial-mesenchymal transitions (EMT) are complex cellular programs that were repeatedly implicated in cancer cell invasion and metastasis (4). In the course of EMT, cells gradually trade key epithelial characteristics such as apical-basal polarity, and tight cell-cell and cell-matrix contacts for mesenchymal features including fibroblast-like morphology, frontrear polarity, increased motility, and enhanced invasiveness. Cancer cells can be induced to undergo EMT in response to extrinsic and intrinsic stimuli, for example from the Wnt, Notch, mitogen-activated protein kinase (MAPK), and TGF $\beta$ signaling pathways (4). Irrespective of the upstream trigger, a central role in EMT processes is typically attributed to a small group of transcription factors (TFs) from the Snail, Zeb, and Twist families (5). These EMT-TFs are thought to orchestrate the transition from epithelial to mesenchymal states by extensive transcriptional reprogramming. Yet, recent studies suggest that cancer cells may not need to acquire a fully mesenchymal phenotype in order to attain maximum invasive and metastatic capacity. Rather, the ability of cancer cells to traverse only partway through EMT and to present with variable combinations of epithelial and mesenchymal properties appears to be most advantageous for metastasis $(6,7)$. Although instances of such partial EMT (pEMT) 
could be captured in vitro and in vivo $(6,8-12)$, it is not clear whether pEMT states only represent snapshots along a continuum of intermediate states towards complete EMT (cEMT) $(8,12)$, or endpoints of independent pEMT programs. Furthermore, it also became evident that tissue-specific and transcription-independent variants of pEMT exist $(9,10)$.

Colorectal cancer $(\mathrm{CRC})$ is one of the most frequent forms of cancer worldwide. Histological examination indicated that the predominant form of stromal infiltration in $\mathrm{CRC}$ is collective invasion with evidence for pEMT at the invasive front (3). How this particular pattern of invasiveness arises is unknown. Work with genetically engineered mouse models and organoids suggested that the three most common tumor-promoting events in CRC, disruption of the Apc and Trp53 tumor suppressor genes in conjunction with oncogenic mutations in Kras, suffice to induce invasion and metastatic disease (13-16), but it was not determined whether the experimental models recapitulated the particular type of invasion observed in CRC tissue specimens. Furthermore, there are contradictory results concerning the number and type of genetic changes needed to elicit invasion and metastasis $(15,17-$ 20), and an invasion-stimulating effect of the tumor microenvironment (TME) cannot be excluded.

TGF $\beta$ family members are prototypic examples for microenvironmental factors with the capacity to induce cancer cell invasion. However, the role of TGF $\beta$ signaling in CRC is quite controversial. TGF $\beta$ receptors and Smad4, a key TF in canonical TGF $\beta$ signaling, are frequently inactivated to boost experimental metastasis in animal models $(15,17-21)$, and it was proposed that TGF $\beta$ signaling might act on nonneoplastic rather than cancer cells to promote metastases formation $(22,23)$. Yet, the TGF $\beta$ pathway appears mostly functional in CRC specimens (24), and comprehensive transcriptome analyses provided evidence for active TGF $\beta$ signaling in the consensus molecular subtype 4 (CMS4) of human CRC, which is the CMS with the poorest prognosis (25). Likewise, TGF $\beta$ pathway activity was 
documented in a metastatic mouse tumor model (13), and TGF $\beta$ signaling induced invasion and installed CMS4-like transcription in a model of the sessile serrated adenoma CRC subtype (26). Even though TGF $\beta$ signaling typically induces cEMT and single cell invasion $(8,10,12,27)$, it therefore could be that invasive behavior in CRC is dual a consequence of cell-autonomous and non-autonomous mechanisms.

Here, we aimed to dissect the impact of tumor cell genetics and extrinsic factors on invasive behavior in intestinal tumorigenesis by employing genetically modified murine organoids under defined conditions. We report that disruption of Apc and Smad4 together with expression of $\mathrm{Kras}^{\mathrm{G} 12 \mathrm{D}}$ and the dominant negative $\mathrm{p} 53^{\mathrm{R} 172 \mathrm{H}}$ mutant do not suffice to elicit cellintrinsic invasiveness. However, concomitant oncogenic lesions in the Wnt, MAPK, and p53 signaling pathways primed organoids for TGF $\beta 1$-inducible collective invasion and a stable, transcription-dependent pEMT which was executed independently of Snail1 and Zeb1. Interestingly, TGF $\beta 1$-treated organoids acquired a gene expression pattern representative of CMS4 suggesting that an atypical TGF $\beta 1$ response and a novel pEMT variant may underlie human $\mathrm{CRC}$ collective invasion. 


\section{Results}

\section{Intestinal organoids do not gain cell-intrinsic invasiveness by oncogenic}

transformation. To test whether hyperactive Wnt and MAPK signaling and impaired p53 activity induce invasiveness in a cell-autonomous fashion, we generated multiple small intestinal organoid lines from $A p c^{580 S / 580 S}, \mathrm{Kras}^{L S L-G 12 \mathrm{D} /+}, \operatorname{Trp}^{\mathrm{LSSL-R172H/+}}, \operatorname{tg}^{\mathrm{LV} \text { VillinCreER }} \mathrm{R}^{\mathrm{T2}}$ mice (Fig. 1a, Supplementary fig. 1). Organoids (hereafter termed floxed organoids) were treated with 4-hydroxy-tamoxifen (4-OHT) in vitro to obtain Apc-deficient organoids expressing oncogenic Kras ${ }^{\mathrm{G} 12 \mathrm{D}}$ and dominant negative $\mathrm{p} 53^{\mathrm{R} 172 \mathrm{H}}$ (hereafter called TKA organoids; Supplementary fig. 1a-d). Floxed and TKA organoids were analyzed for evidence of oncogenic transformation and invasiveness. As observed before $(17,18)$, TKA organoids exhibited cystic growth when compared to bud-forming floxed organoids (Fig. 1b) and remained viable in media without R-spondin-1 (Supplementary fig. 1e, f). TKA organoids were also EGF-independent and tolerated EGFR inhibition (Supplementary fig. 1e, f). Furthermore, expression of Wnt target genes and intestinal stem cell markers increased in TKA organoids while that of differentiation markers decreased (Supplementary fig. 1g). This suggests an expansion of stem and progenitor cells in TKA organoids at the expense of multilineage differentiation. Notably, acquired growth factor independence and disturbed differentiation represent hallmarks of cancer cells. Yet, TKA organoids remained noninvasive. Like floxed organoids, they were surrounded by a continuous layer of laminin and showed apical localization of atypical protein kinase C (aPKC) (Fig. 1c). Forskolin-inducible swelling of organoids confirmed epithelial integrity (28), albeit floxed and TKA organoids displayed different swelling dynamics which might be caused by the differences in shape and elasticity of organoids (Fig. 1d). Thus, TKA organoids maintained apico-basolateral polarity, basement membrane integrity, and functional cell-cell junctions which are key characteristics of epithelial cell layers. There was also no indication for invasiveness when organoids were cultivated in an air-liquid interface setup with type I collagen to expose them to an extracellular matrix (ECM) more representative of a desmoplastic tumor microenvironment (29). The majority of non-transformed floxed organoids perished under these conditions, 
whereas TKA organoids remained viable and occasionally showed evidence of dysplasia (Fig. 1e). However, TKA organoids did not infiltrate the type I collagen matrix. In summary, despite several lines of evidence for oncogenic transformation, TKA organoids did not display cell-intrinsic invasiveness arguing that invasive behavior observed in vivo might be triggered by cell non-autonomous mechanisms.

TGF $\beta 1$ triggers collective invasion of TKA organoids and ECM remodeling. TGF $\beta 1$ is a prototypical tumor microenvironmental signal and prime inducer of EMT. Because the vast majority of human metastatic CRCs possess an intact TGF $\beta$ signaling machinery (Supplementary fig. 2a), we investigated the cellular and molecular consequences of treating organoids with TGF $\beta 1$. Whereas floxed organoids died when exposed to TGF $\beta 1$, TKA organoids were resistant to TGF $\beta 1$-induced cell death (Supplementary fig. 2b), probably due to expression of oncogenic $\mathrm{Kras}^{\mathrm{G} 12 \mathrm{D}}$ (30). Although levels of cleaved caspase-3, indicative of ongoing apoptosis, increased in TGF $\beta 1$-treated TKA organoids, they were even higher in solvent-treated organoids, and cleaved caspase- 3 was detectable only in cells that had been shed into the lumen of solvent or TGF $\beta 1$-treated TKA organoids (Supplementary fig. 2c, d). Therefore, TGF $\beta 1$ does not appear to cause cell death in TKA organoids. Rather, TGF $\beta 1$ triggered massive morphological changes of TKA organoids which progressively lost their cystic shape, flattened, and extended multicellular protrusions, culminating in the formation of large, cohesive cell sheets and extended strands of cells which infiltrated the surrounding Matrigel (Fig. 2a; Supplementary movie 1). Boyden chamber assays independently confirmed acquired invasiveness of TGF $\beta 1$-stimulated TKA organoids (Fig. 2b, c). TKA organoids embedded in a type I collagen matrix and exposed to TGF $\beta 1$ also remained viable, changed their shape, and became invasive (Fig. 2d). Moreover, colon-derived TKA organoids responded to TGF $\beta 1$ treatment with highly similar morphological changes and also displayed a collective mode of invasion (Supplementary fig. 2e-g). 
Dual fluorescent labelling with membrane-bound mTomato and nuclear H2B-GFP and confocal microscopy revealed that TGF $\beta 1$-treated TKA organoids adopted a dome-like structure with a central lumen and a broad circumferential rim of cells which coherently expanded outwards (Fig. 3a). Single cell delamination was not observed. Invading cell sheets formed only at the organoid base close to the surface of the cell culture plates. Cells at the invasive organoid perimeter produced spike-like fibers of fibronectin [involved in ECM assembly (31)], showed punctiform staining of the integrin $\beta 3$ subunit [involved in adhesion to fibronectin (32)], and of vinculin [marking focal adhesions (33)] (Fig. 3b; Supplementary fig. 3). The observed topological restriction of invasion and formation of fibronectin spikes contrasts with the nuclear localization of Smad2/3 and, hence, active TGF $\beta$ signaling, and expression of fibronectin also in organoid cells constituting the dome structure (Supplementary fig. 4). This suggests that invasive behavior aside from TGF $\beta$ pathway activation additionally depends on a permissive microenvironment. Indeed, to allow uniform TGF $\beta 1$-induced phenotypic switching in organoid cultures we had to lower the Matrigel concentration. Conversely, organoid cells contracted the surrounding ECM while infiltrating: TGF $\beta 1$-treated TKA organoids grown in a type I collagen matrix promoted the formation of larger and more parallel aligned collagen bundles which could be visualized by picrosirius red staining (Fig. 3c). Altogether, TGF $\beta 1$ turned out to promote collective invasion of oncogenically transformed intestinal organoids which appears to involve reciprocal organoid/ECM interactions and ECM remodeling.

\section{Canonical TGF $\beta$ signaling induces $\mathrm{pEMT}$ in oncogenically transformed organoids.}

Classically, TGF $\beta$ signaling induces complete EMT and single cell invasion $(8,10,12,27)$. To better understand TGF $\beta 1$-driven collective invasion of TKA organoids, we performed time-resolved gene expression analyses. TGF $\beta 1$ markedly induced expression of EMT TFs (Snail1, Zeb1), mesenchymal markers (fibronectin/Fn1, N-cadherin/Cdh2) (Fig. 4a, b), and Itga5, which, like Itgb1, is known to be upregulated during EMT (34). Significantly, integrin $\alpha 5 \beta 1$ is a fibronectin receptor (32) and promotes cancer cell migration and invasion (34-36). 
Western blot detection of phosphorylated Smad2/3 additionally confirmed TGF $\beta$ pathway activation. Surprisingly, TGF $\beta 1$ treatment did not diminish but rather increased expression of epithelial markers (E-cadherin/Cdh1, Ephb3, Foxa1), (Fig. 4a, b). Lastly, colonic TKA organoids responded to TGF $\beta 1$ treatment in a virtually identical fashion, showing concomitant upregulation of mesenchymal and epithelial gene expression and elevated levels of EMT-related integrins (Supplementary fig. 5a, b).

Aside from transcriptional repression, a change in intracellular localization of cell-cell adhesion molecules may also contribute to EMT (10). However, immunofluorescence stainings showed that the adherence junction proteins E-cadherin and $\beta$-catenin were retained at cell-cell borders throughout TGF $\beta 1$-treated TKA organoids (Fig. 4c, Supplementary fig. 5c). Only cells at the invasive front showed some increase in cytoplasmic E-cadherin and $\beta$-catenin staining, delocalization of the tight junction protein claudin-7, and reduction of aPKC (Fig. 4c). Despite these graded phenotypic changes, we conclude that TGF $\beta 1$-stimulated TKA organoids adopt a pEMT state distinguished by concurrent exhibition of epithelial and mesenchymal characteristics and largely maintained membranous Ecadherin and $\beta$-catenin.

The unexpected induction of pEMT by TGF $\beta 1$ prompted us to investigate the underlying signal transduction mechanisms. Overexpression of constitutively active TGF- $\beta$ receptor type 1 (TGFBR1CA) (37) fully recapitulated TGF $\beta 1$-induced morphological changes and invasive behavior of TKA organoids (Supplementary fig. 6a-c). Conversely, expressing dominant negative TGF- $\beta$ receptor type 2 (TGFBR2DN) (38), and pharmacologically inhibiting TGFBR1 blocked TGF $\beta 1$-induced phenotypic alterations (Fig. 5a-d, Supplementary fig. 6d). Finally, we knocked out Smad4, and generated quadruple mutant TKAS organoids (Fig. 5e-g). Unlike one might have expected $(15,17,18,21)$, Smad4-deficiency did not promote invasiveness per se but abrogated the TGF $\beta 1$ response (Fig. 5h-j, Supplementary 
fig. 6e). We conclude that the observed pEMT of oncogenically transformed intestinal organoids is conferred by receptor-mediated, canonical TGF $\beta$ signaling.

\section{Transcriptomes of TGF $\beta 1$-treated TKA organoids resemble CMS4 of human colorectal}

cancer. To comprehensively characterize TGF $\beta 1$-induced pEMT, we performed timeresolved transcriptome analysis by RNA sequencing (RNA-seq). Organoids were stimulated with TGF 31 for up to $72 \mathrm{~h}$ or harvested at the onset of the experiment $(\mathrm{C}-0)$ and after $72 \mathrm{~h}$ of cultivation with solvent (C-72) to account for culture-dependent effects. Principal component (PC) analysis revealed high concordance between independent biological replicates from two organoid lines (Fig. 6a). A clear separation of control and TGF $\beta 1$-stimulated samples occurred along PC1, which accounts for most of the variances and likely reflects TGF $\beta 1$ induced gene expression changes over time. An additional segregation of samples along PC2 might be attributable to organoid maturation during cultivation (Fig. 6a). TGF $\beta 1$ caused extensive transcriptome changes eventually comprising 2,349 upregulated genes and 2,471 downregulated genes after $72 \mathrm{~h}$ of stimulation (Fig. 6b, Supplementary table 2; adjusted pvalue $<0.01, \mid \log _{2}($ fold change $\left.[F C]) \mid>1\right)$. Functional enrichment analyses of gene sets revealed gene ontology (GO) terms related to cell-cell adhesion, locomotion, extracellular structure organization, integrins, focal adhesion, and collagen formation as significantly enriched among genes upregulated by TGF $\beta 1$ (Fig. 6c). In agreement with this and with the observed changes in invasiveness, ECM deposition, and reorganization, the RNA-seq data showed TGF $\beta 1$-mediated elevated expression of ECM components, receptors, and remodeling enzymes (Supplementary table 3). Genes downregulated by TGF $\beta 1$ were enriched for GO terms connected to RNA and protein metabolic processes, DNA replication, and cell cycle (Supplementary fig. 7a). Indeed, compared to controls, TGF $\beta 1$-treated TKA organoids contained only few Ki67-positive cells which were concentrated in the organoid center (Supplementary fig. 7b). Accordingly, reduced proliferation may represent a common feature of partial and complete EMT (39). 
Additionally, we related our transcriptome data to previously defined EMT gene expression signatures (40-44). Published data sets were split into epithelial and mesenchymal components, and enrichment of sub-signatures among the TGF $\beta 1$ up- and downregulated genes in TKA organoids was examined. Notably, TGF $\beta 1$-upregulated genes were significantly enriched for all mesenchymal and even two epithelial sub-signatures (Fig. 6d), while TGF $\beta 1$-downregulated genes showed no enrichment at all, confirming on a larger scale that TGF $\beta 1$-stimulated TKA organoids chiefly retain epithelial gene expression while concomitantly acquiring a mesenchymal transcriptional profile.

Human colorectal tumors can be classified into four subgroups CMS1-4 with distinctive transcriptomic features (25). To assess whether TKA organoids could be allocated to any of these, all RNA-seq data sets from control and TGF $\beta 1$-treated TKA organoids were compared to the CMS transcriptional profiles. Control organoids (C-0) and organoids treated with TGF $\beta 1$ for $6 \mathrm{~h}$ could not be classified (Fig. 6e), perhaps because mechanical disruption and reseeding of organoids had erased any typifying gene expression. The C-72 control group possessed gene expression properties of CMS2 which agrees well with hyperactive Wnt signaling in Apc-deficient TKA organoids (25). In contrast, past $24 \mathrm{~h}$ of TGF $\beta 1$ treatment, TKA organoids showed a uniform association with CMS4 which is defined as mesenchymal with signs of increased TGF $\beta$ signaling and EMT (25). Notably, when we interrogated human colon cancer transcriptome data from The Cancer Genome Atlas (TCGA) with respect to enrichment of epithelial and mesenchymal EMT sub-signatures, we found that genes upregulated in CMS4 compared to CMS2 were significantly enriched for all mesenchymal sub-signatures. Again, downregulated genes displayed no enrichment for any sub-signature (Fig. 6f). This further highlights the similarity between transcriptomes of TGF $\beta 1$-treated TKA organoids and human CMS4 tumors, and additionally hints that CMS4 tumors likewise exhibit a pEMT. 
Next, we used ISMARA (Integrated System for Motif Activity Response Analysis) (45) to deduce from RNA-seq data potential regulatory DNA sequence motifs and TFs, whose activity might drive pEMT of TGF $\beta 1$-treated TKA organoids. Motifs identified by ISMARA were ranked according to their z-values as a measure for the contribution of each motif to the observed transcriptional pEMT program. Top entries for motifs with increased activity upon TGF $\beta 1$ treatment pointed to an involvement of Sox proteins, Sp1, Krüppel-like factors, Tead3/4, Snail1, Snail2, Zeb1, Fos and Jun family members, Smad4, and the Ets TF family (Supplementary table 4), all of which were implicated in EMT processes before $(4,7)$. DNA sequence motifs with reduced activity refer among others to the Nfy, E2f, and Myb families of cell cycle regulators which fits to the TGF $\beta 1$-induced decrease in proliferation in organoids.

We further compared ISMARA results to a collection of DNA sequence motifs which were previously implicated in shaping transcriptional landscapes at different stages of EMT (6). Activities of DNA sequence motifs common to all EMT states (Jun, Ets, Runx, Nfi, Tead3/4) were slightly higher under TGF $\beta 1$-treatment compared to controls and remained largely constant over time (Supplementary fig. 8a, b), except for the Tead3/4 motif which exhibited a large net difference in activity due to strongly decreased activity under control conditions. Possibly, this results from organoid maturation. In contrast to elevated and constant activities of pan-EMT state motifs, DNA sequence motifs which had previously been associated with distinct epithelial, mesenchymal and intermediate EMT states in squamous cell carcinoma and breast cancer (6), presented mostly with low z-values and only small activity changes, especially when taking into account changes in motif activity related to cultivation time (Supplementary fig. 8c). This difference in DNA sequence motif activity argues that alternative sets of TFs drive EMT processes in intestinal cancer versus other tumor entities. This notwithstanding, constant and increasing activities of motifs which were reported to be active specifically in epithelial cancer cells (Sox2, Trp63, Grhl1, Tfap2c) and early and late pEMT states (Sp1, Trp63, Tfap2c), further support that TGF $\beta 1$-induced pEMT of TKA 
organoids is distinguished by a reinforcement of epithelial features along with the additional gain of mesenchymal characteristics.

TGF $\beta 1$-induced pEMT occurs independently from EMT master regulators. Snail, Zeb, and Twist TF family members are thought to fulfil key functions in EMT processes $(4,46)$. Since Snai1 and Zeb1 were the only EMT-TF genes upregulated in TGF $\beta 1$-treated TKA organoids (Supplementary table 2), we examined their roles in TGF $\beta 1$-inducible pEMT. Both genes were targeted applying a dual sgRNA-mediated deletion strategy, and multiple, clonally derived wildtype (TKA-Snai $1^{\mathrm{wt}}$, TKA-Zeb $1^{\mathrm{wt}}$ ) and knockout (TKA-Snai ${ }^{\mathrm{KO}}$, TKA$\mathrm{Zeb}^{\mathrm{KO}}$ ) organoid lines were obtained (Fig. 7a). Importantly, Snail1 and Zeb1 deficiencies did not impair TGF $\beta$ pathway activity as demonstrated by unabated Smad2/3 phosphorylation (Fig. 7b). Despite complete absence of Snail1 and Zeb1, however, TGF $\beta 1$-regulated expression of EMT-associated genes, as well as TGF $\beta 1$-induced morphological conversion and invasiveness were unaffected (Fig. 7b; Supplementary fig. 9a, b). To complement these loss-of-function experiments, we generated TKA organoids with doxycycline (Dox)-inducible expression of Snail1 and ZEB1 (Supplementary fig. 10a, b). While ZEB1 overexpression had no discernible impact on organoid morphology and invasiveness (Supplementary fig. 10c-e), TKA organoids expressing Snail1 lost their cystic shape, occasionally infiltrated the surrounding Matrigel, and exhibited some invasiveness in Boyden chamber assays, albeit much less pronounced compared to TGFß1 stimulation (Supplementary Fig. 10c-e). Neither ZEB1 nor Snail1 overexpression reproduced TGF 1 1-mediated gene expression changes (Supplementary fig. 10f). All-in-all, overexpression of Snail1 and ZEB1 did not mimic the TGF $\beta 1$ response of TKA organoids. Thus, classical EMT-TFs are neither sufficient nor required for TGF $\beta 1$-mediated pEMT and collective invasion. 


\section{Discussion}

To decipher the molecular and cellular basics of tumor invasion and metastasis poses a persistent challenge, and it is not clear to which extent these processes are driven by genetic changes in cancer cells and by extrinsic factors from their surroundings. Here we used small intestinal and colonic organoids in a naïve, wildtype state to inflict oncogenic lesions only in vitro. This allowed us to assess the cell-autonomous impact of oncogenic transformation on epithelial integrity and invasiveness without prior exposure to any confounding influence from non-tumor tissue as e. g. in animal models. Under these circumstances, we found that the concomitant introduction of up to four mutations in Apc, Kras, Trp53, and Smad4 was insufficient to elicit cell-intrinsic invasive behavior in organoids. This contrasts with results from genetically-engineered mouse models and organoid transplantation experiments where similar combinations of mutations led to the formation of invasive (since metastasizing) tumors (13-21). This difference in invasiveness in vitro and in vivo strongly argues that stimuli from the microenvironment were responsible for triggering tumor cell invasion in the in vivo settings. In this sense, oncogenic transformation constitutes an obligatory prerequisite, but only conditions tumor cells to become responsive to external pro-invasive signals. The implication of cell non-autonomous mechanisms in metastasis is consistent with the genetic similarity between primary and secondary lesions, and the failure to identify dedicated metastasis driver and suppressor genes in CRC (47). Furthermore, the critical importance of the tumor microenvironment for invasion and metastasis could readily explain inconsistent results concerning the mutational spectrum required for metastasis in autochthonous tumor models and upon heterotopic transplantations (13-21).

To test the idea that microenvironmental signals elicit invasiveness of oncogenically transformed organoids, we used the TGF $\beta$ pathway as a proof-of-principle. TGF $\beta$ signaling was previously shown to promote intestinal cancer metastasis, albeit indirectly by acting on non-cancer cells $(22,23)$. Although it is commonly pointed out that TGF $\beta$ pathway components are frequently mutated in colorectal tumors, and that disruption of TGF $\beta$ 
signaling promotes malignant progression in experimental models of intestinal cancer (15, 17-21) our results show that cancer cells themselves can be relevant targets of TGF $\beta$ signaling. In support of this, TGF $\beta$ receptors and SMAD genes are intact in more than $60 \%$ of human CRCs (24), and TGF $\beta$ pathway activity is evident in CMS4 cancers (25).

Our findings reinforce the importance of the tumor microenvironment for tumor invasion (48, 49). They further demonstrate that the tumor microenvironment can influence cancer cells in different, yet cooperating ways, first by providing an invasion-permissive ECM and second by supplying pro-invasive growth factors and cytokines. Nonetheless, oncogenically transformed organoids did not appear to just follow environmental cues, but actively participated in reciprocal interactions with their surroundings. The capacity to deposit and remodel ECM components in the tumor stroma therefore may not be restricted to cancer-associated fibroblasts and other non-cancer cells $(50,51)$.

TGF $\beta$ ligands are paradigmatic inducers of EMT through extensive transcriptional reprogramming which engages EMT-TFs and typically culminates in complete mesenchymal conversion $(8,10,12,27)$. Therefore, the ability of canonical, Smad4-dependent TGF $\beta$ signaling to induce pEMT without progression to a fully mesenchymal state represents a novel finding. Additionally, pEMT in TKA organoids was accompanied by collective invasion which likewise is not commonly associated with TGF $\beta$-induced EMT $(4,5,10,27)$. Moreover, collective invasion and pEMT of TKA organoids were transcriptionally regulated whereby a mesenchymal gene expression program was implemented on top of a largely maintained epithelial program, including chiefly sustained membrane localization of E-cadherin and $\beta$ catenin. The hybrid epithelial/mesenchymal state of TGF $\beta 1$-treated TKA organoids therefore differs from previously described variants of pEMT which involved broadly downregulated epithelial gene expression or the abrogation of epithelial cell characteristics by transcriptionindependent internalization of cell adhesion molecules $(6,10)$. Interestingly, the gene regulatory network which was activated by TGF $\beta 1$ in TKA organoids, seemingly differs from 
that operating in pEMT states in squamous cell carcinoma and breast cancer $(6,11)$. Accordingly, we believe that the TGF 11 response of oncogenically transformed intestinal cells represents a new and distinct pEMT program.

We suspect that the switch from TGF $\beta 1$-induced complete to partial EMT which we observed, is a consequence of context-dependent differences in the functionality of EMTTFs. Not only were Snail1 and Zeb1 dispensable for TGF $\beta 1$-induced pEMT, but also overexpression experiments revealed much restricted EMT-inducing capacities of the two factors. Impaired function of EMT-TFs could be related to peculiarities of intestinal cells concerning posttranslational modifications of Snail1 and Zeb1 and to the expression of transcriptional co-factors (46). Above that, our results imply that EMT-TFs can be upregulated as passengers without functionally contributing to EMT processes. Conversely, lack of EMT-TF expression may not equal absence of EMT. This has implications for the use of Snail1 and Zeb1 as EMT markers, and for the interpretation of findings which refuted a role of EMT in metastasis based on knockout animals (52).

In agreement with their genetic constitution, TKA organoids exhibited a gene expression pattern resembling CMS2 which is dominated by Wnt pathway activity (25). Upon treatment with TGF $\beta 1$, organoids adopted a transcriptional profile highly similar to the transcriptome of CMS4 cancers which indeed are characterized by TGF $\beta$ pathway activation, mesenchymal features, high stromal content, and particularly poor prognosis (25). Convertibility to CMS4, thus, may not be limited to the sessile serrated adenoma path of colorectal carcinogenesis (26). Acquisition of CMS4 characteristics may only require a functional TGF $\beta$ pathway and could be determined primarily by microenvironmental influences. Notably, re-examination of gene expression patterns unearthed evidence for pEMT in human CMS4 samples, strengthening the significance of our findings for human cancer. Adding further to the pathophysiological relevance of our study, we note that collective invasion, which was 
triggered by TGF $\beta 1$ in TKA organoids, accurately reflects the predominant mode of stromal infiltration in most carcinomas.

Altogether, our study significantly expands the understanding of the mechanistic foundations of partial EMT and its context-dependent, distinctive appearances. Thereby, it may point out potential therapeutic strategies to eventually interfere with the metastatic cascade.

\section{Acknowledgements}

The authors are grateful to the team of the Genomics and Proteomics Core Facility, German Cancer Research Center/DKFZ, Heidelberg, Germany, for next generation sequencing services, and to the staff of the Life Imaging Center (LIC) at the Center for Biological Systems Analysis (ZBSA) of the Albert-Ludwigs-University Freiburg for help with confocal microscopy. We especially appreciate the excellent support in image recording and analysis. We thank the members of the Hecht laboratory for helpful discussions and critical reading of the manuscript. We are particularly indebted to Vivien Freihen for her invaluable advice and initial introduction to the cultivation and handling of intestinal organoids. This study was supported in part by the Excellence Initiative of the German Research Foundation (GSC-4, Spemann Graduate School) and in part by the Ministry for Science, Research and Arts of the State of Baden-Wuerttemberg. Additional financial support was obtained from the Deutsche Forschungsgemeinschaft (DFG) (CRC-850 subprojects $B 5$ to $A H, B 11$ to $A N$, and $Z 1$ to $M B$ ), and from the German Federal Ministry of Education and Research (BMBF) within the framework of the e:Med research and funding concept CoNfirm (FKZ 01ZX1708F to MB).

\section{Author Contributions}

$\mathrm{MF}, \mathrm{AN}, \mathrm{MB}$, and $\mathrm{AH}$ conceived and designed experiments. MF and MS generated data and analyzed all aspects of organoid morphology, histology, invasive behavior, and gene expression, and did the CRISPR/Cas9 genome editing experiments. MF and AN analyzed ECM remodeling by invasive organoids, acquired microscopic images and quantified 
collagen fiber formation. SD and MB carried out the bioinformatic analyses of the RNA-seq data, including identification of differentially expressed genes, gene set enrichment analyses, and $\mathrm{CMS}$ classification. MF, SD, MB, and $\mathrm{AH}$ interpreted data, created figures and wrote the manuscript. All authors critically read and commented on the final version of the manuscript.

\section{Competing interests statement}

The authors declare no competing interests. 


\section{Materials and methods}

Organoid culture. Small intestinal and colonic organoid lines were established from C57BL/6N Apc ${ }^{580 S / 580 S} ; \mathrm{Kras}^{L S L-G 12 D /+} ; \operatorname{Trp53}^{L S L-R 172 H /+} ; \operatorname{tgVillin-CreER^{T2}}$ mice (53-56) as described (57), and labeled with the identifier of founder animals. Mice were handled in accordance with legal regulations at the Center for Experimental Models and Transgenic Service of the University of Freiburg Medical Center (project registration number: X-17/07S). To recombine conditional alleles, organoids were treated with $0.5 \mu \mathrm{M}$ 4-hydroxy-tamoxifen (4-OHT; Sigma Aldrich, H7904) for up to 120 h. Recombination was verified by PCR with primers listed in Supplementary table 5 and genomic DNA isolated with the ReliaPrep ${ }^{\mathrm{TM}}$ gDNA Tissue Miniprep System (Promega, A2052) and peqGOLD Tissue DNA Mini Kit (Peqlab, 12-3496-02). Upon inactivation of Apc, R-spondin-1 was omitted from the culture media.

Manipulation of TGF $\beta$ and EGF signaling. For experiments involving TGF $\beta$ signaling, organoids were mechanically disrupted and seeded 30-40 h before treatments were started. To activate and inhibit TGF $\beta$ signaling, $5 \mathrm{ng} / \mathrm{ml}$ human TGF $\beta 1$ (Peprotech, 100-21) and $10 \mu \mathrm{M}$ SB431542 (Selleckchem, S1067) were administered, respectively. To assess independence from EGFR signaling, organoids were mechanically disrupted and seeded in Matrigel two days before treatment with $0.8 \mu \mathrm{M}$ Gefitinib (Selleckchem, S1025) for $72 \mathrm{~h}$. Organoid viability was then determined by phase contrast microscopy and subsequent incubation with $500 \mu \mathrm{g} / \mathrm{ml}$ MTT (3-(4,5-dimethylthiazol-2-yl)-2,5-diphenyltetrazolium bromide) at $37^{\circ} \mathrm{C}$ for $1 \mathrm{~h}$. Culture plates were imaged with a CanoScan 9950F scanner (Canon). Culture media supplemented with growth factors, inhibitors and other reagents were refreshed every $48 \mathrm{~h}$. 
Assessment of epithelial integrity. To test for epithelial integrity, organoids were mechanically disrupted and seeded in Matrigel two days before treatment with $5 \mu \mathrm{M}$ forskolin (Selleckchem, S2449). Swelling was monitored for $8 \mathrm{~h}$ at 20 min intervals using the JuLI ${ }^{\mathrm{TM}}$ Stage Real-Time Cell History Recorder (NanoEntek) and a 10x objective.

Viral transduction. Lentiviral and retroviral particles were produced by co-transfecting HEK293T cells with viral vectors and packaging plasmids at a mass ratio of 1:0.75:0.3 using FuGENE®6 (Promega, E2691). Virus-containing medium was filtrated $(0.45 \mu \mathrm{m}) 48 \mathrm{~h}$ posttransfection and centrifuged at $8,000 \mathrm{~g}$ and $4^{\circ} \mathrm{C}$ overnight. Viral pellets were resuspended in culture medium supplemented with $8 \mu \mathrm{g} / \mathrm{ml}$ Polybrene (Merck, TR-1003-G) and $10 \mu \mathrm{M} \mathrm{Y}$ 27632 (Selleckchem, S1049). For infection, organoids were dissociated into single cells by incubation with Accutase at $37^{\circ} \mathrm{C}$ for $10 \mathrm{~min}$, mixed with the viral suspensions, plated on a Matrigel bed, and incubated at $37^{\circ} \mathrm{C}$ for $8-9 \mathrm{~h}$. After washing with PBS, attached cells were overlaid with Matrigel and provided with culture medium containing Y-27632. Floxed organoids additionally received $1 \mu \mathrm{M}$ valproic acid (Sigma Aldrich, P4543) and $1 \mu \mathrm{M}$ CT99021 (Selleckchem, S2924). Selection with antibiotics was started three days (floxed organoids) and two days (TKA organoids) after transduction, using $5 \mathrm{ng} / \mathrm{ml}$ blasticidin (Invitrogen, R210-01), $2 \mu \mathrm{g} / \mathrm{ml}$ puromycin (Sigma Aldrich, P7255), $500 \mu \mathrm{g} / \mathrm{ml}$ geneticin (G418; Thermo Fisher Scientific, 10131027) (floxed organoids), and $700 \mu \mathrm{g} / \mathrm{ml}$ G418 (TKA organoids). For Dox-inducible gene expression, organoids were transduced with pMSCVrtTA2-PGK-eGFP-F2A-NeoR, followed by a second infection with pRetroX-tight-Snail1-HAPuroR, pRetroX-tight-ZEB1-HA-PuroR, or pRetroX-tight-MCS-PuroR $(58,59)$. Expression of Snail1 and ZEB1 was induced with $1 \mu \mathrm{g} / \mathrm{ml}$ Dox (Sigma Aldrich, D9891). For 4-OHTinducible gene expression, TKA organoids were transduced with pMSCV-loxP-BlastR-loxPTGFBR1(T204D)-F2A-eGFP (coding for TGFBR1CA), pMSCV-loxP-BlastR-loxPTGFBR2( $\triangle$ cyt)-F2A-eGFP (coding for TGFBR1DN), and pMSCV-loxP-BlastR-loxP-eGFP. Expression of TGF $\beta$ receptor mutants was induced by treating organoids with $0.5 \mu \mathrm{M} 4-\mathrm{OHT}$. In case of TGFBR2DN, this was done three days prior to the start of invasion and gene 
expression experiments. For fluorescent labeling and live cell imaging, TKA organoids were transduced with pLenti-SV40-mTomato-P2A-H2B-GFP-PuroR. All constructs used in the study and their parental vectors are listed in Supplementary table 6.

Genome editing. Smad4, Snai1, and Zeb1 were inactivated by frame-shift-inducing exon deletions. For this, suitable exons were targeted by two single guide RNAs (sgRNAs; target sites listed in Supplementary table 5) selected using CCTop (60). Expression cassettes for sgRNAs were generated using vectors from the MuLE system (Supplementary table 6). For Smad4 deletion, $400 \mathrm{ng}$ of each sgRNA expression plasmid and 700 ng pCAG-Cas9-turboRFP vector were transfected into floxed organoids as described (17), except that single cell suspensions were generated with Accutase and transfection was done with Lipofectamine ${ }^{\circledR}$ LTX (Invitrogen, 15338100). Three days after transfection, Smad4-deficient organoids were selected for by adding $100 \mathrm{ng} / \mathrm{ml}$ murine BMP-4 (Peprotech, 315-27) to the culture media while omitting Noggin. Smad4-deficient organoids were treated with 4-OHT to generate quadruple mutant TKAS organoids. To inactivate Snai1, floxed organoids were transduced with pLenti-Cas9-T2A-BlastR and selected with blasticidin. Cas9-expressing organoids were transduced with pLenti-Dest-Snai1-sgRNA1+2-eGFP-F2A-NeoR-loxP and selected with G418 for one week. Thereafter, $0.5 \mu \mathrm{M}$ 4-OHT was administered for 5 days to induce excision of loxP-flanked DNA sequences including the sgRNA-expressing provirus. Organoids were dissociated and sparsely seeded to facilitate clonal outgrowth. Single cellderived organoids were manually picked, expanded, and screened by PCR with primers listed in Supplementary table 5. Zeb1 was inactivated similarly, except that TKA organoids were simultaneously transduced with pLenti-Cas9-T2A-BlastR and pLenti-Dest-Zeb1sgRNA1+2-eGFP-F2A-NeoR-loxP, co-selected with blasticidin and G418, and exposed to 4OHT for 3 days.

Boyden chamber invasion assay. Organoids were mechanically disrupted and seeded into inserts for 24-well plates (Falcon, 353097) with Matrigel $30-40 \mathrm{~h}$ prior to treatments as 
described in the figure legends. Invaded cells on the bottom surface of the membranes were fixed with $4 \%$ paraformaldehyde at room temperature (RT) for 10 min and stained with $0.1 \%$ (w/v) crystal violet in water at RT for $10 \mathrm{~min}$. For quantification, membranes were imaged using the BZ-9000 fluorescence microscope (Keyence) and the proportion of the membranes covered with invasive cells was determined using ImageJ.

Air-liquid interface (ALI) culture. For ALI cultures (19), organoids were incubated in Cell Recovery Solution (Corning, 354253) on ice for $1 \mathrm{~h}$, washed in PBS, mechanically disrupted, and seeded in type I collagen into 24-well transwell filter inserts. After gelation, culture medium was added to the outer well. Medium was exchanged every three days. After ten days, cultures were fixed in $4 \%$ paraformaldehyde at RT overnight, paraffin-embedded, sectioned into $5 \mu \mathrm{m}$ slices, stained with hematoxylin and eosin, and imaged using the BZ9000 fluorescence microscope (Keyence).

Culture in type I collagen. Type I collagen (Collagen I HC, rat tail, Corning, 354249) was diluted with cold PBS to a concentration of $3 \mathrm{mg} / \mathrm{ml}$. 1/20 (v/v) Medium 199 (Sigma Aldrich, M0650) was added, the $\mathrm{pH}$ was adjusted with $\mathrm{NaOH}$, and the matrix was incubated on ice for $1 \mathrm{~h}$. Organoids were prepared as described for ALI cultures, resuspended in the collagen matrix, and seeded on pre-warmed plates. After gelation, culture medium was added. Two days later, treatment with solvent or TGFß1 was started.

Picrosirius red staining. After $96 \mathrm{~h}$ of solvent or TGF $\beta 1$ treatment, organoid cultures in type I collagen were fixed in $10 \%$ formalin at RT overnight, paraffin embedded, and sectioned into $40 \mu \mathrm{m}$ slices. Picrosirius red staining was performed as described (61). Sections were imaged using an Axioplan2 fluorescence microscope (Zeiss) equipped with a polarizer, an analyzer, and an Axiocam camera. All pictures taken with polarized or nonpolarized light were acquired with the same exposure times. To quantify the formation of parallel and larger collagen bundles, images were acquired under polarizing light with a 
$540 \mathrm{~nm}$ filter. After acquisition, picrosirius red-derived signals above a defined threshold were measured within a $65 \mu \mathrm{m}$ wide area immediately surrounding an organoid under investigation, while excluding the organoid itself.

Immunofluorescence staining of paraffin sections and whole mounts. For sectioning, organoids were fixed in $4 \%$ paraformaldehyde overnight at 3-4 days after seeding, paraffinembedded, and sectioned into $5 \mu \mathrm{m}$ slices. Sections were stained as described (62). For whole mount imaging, organoids were seeded into chambered coverslips (Ibidi, 80826), treated with solvent or TGF $\beta 1$ for $72 \mathrm{~h}$, and fixed in situ with $4 \%$ paraformaldehyde at $4^{\circ} \mathrm{C}$ for $45 \mathrm{~min}$. After permeabilization with $0.5 \%(\mathrm{v} / \mathrm{v})$ Triton $\mathrm{X}-100$ in PBS at $4{ }^{\circ} \mathrm{C}$ for $10 \mathrm{~min}$ and quenching with $0.1 \mathrm{M}$ glycine in PBS at RT for $15 \mathrm{~min}$, samples were incubated with blocking buffer (PBS with 10\% FCS, 0.2\% Triton X-100, 0.05\% Tween20, 0.1\% [w/v] BSA) at RT for 1 h. Primary antibodies were diluted in blocking buffer and incubated at $4^{\circ} \mathrm{C}$ overnight. After washing with blocking buffer, organoids were incubated with $0.3 \mu \mathrm{M}$ DAPI and secondary antibodies diluted in blocking buffer. For actin staining, $5 \mathrm{u} \mathrm{ml}^{-1}$ phalloidin CF555 (Biotium, 00040) were added together with the secondary antibodies. After washing with blocking buffer, organoids were mounted with $0.1 \%(w / v)$ n-propyl gallate (Sigma Aldrich, P3130) in PBS. Primary and secondary antibodies are listed in Supplementary table 7.

Fluorescence microscopy. Images of immunofluorescence stainings of paraffin sections were acquired using an Axio Observer.Z1 fluorescence microscope with an ApoTome2 equipment (Zeiss). Whole mount immunofluorescence stainings were imaged with a LSM 880 confocal microscope (Zeiss) with an Achroplan IR 40x/0.8 W objective and laser wavelengths of 405,488 , and $561 \mathrm{~nm}$, unless stated differently. From image stacks $(1.04 \mu \mathrm{m}$ step size) orthogonal views of cross sections were generated using ZEN 2.3 and ImageJ. For live imaging, TKA organoids expressing mTomato and H2B-GFP were seeded into chambered culture plates (Ibidi, 80416). During confocal microscopy (settings as above), organoids were kept at $37^{\circ} \mathrm{C}$ and $5 \% \mathrm{CO}_{2}$ in a Tokai Hit Incubator. Orthogonal views of cross 
sections and $3 \mathrm{D}$ reconstructions from live imaging data were generated using Huygens Professional for deconvolution and Imaris.

RNA isolation, qRT-PCR, and RNA-seq. RNA was isolated and cDNA was synthesized with the peqGOLD MicroSpin Total RNA Kit (Peqlab/VWR Life Science, 12-6831) and the qScript $^{\mathrm{TM}}$ Flex cDNA Kit (Quantabio, 95049), respectively. For qRT-PCR, the PerfeCTa® SYBR® Green SuperMix (Quantabio, 95049) was employed (primers listed in Supplementary table 8) with amounts of cDNA equivalent to $10 \mathrm{ng}$ and $20 \mathrm{ng}$ RNA when PCRs were conducted in a CFX384 and CFX96 Touch Real-Time PCR Detection System, respectively, (Bio-Rad Laboratories). Following normalization to Gapdh or Eef1a1 transcripts, gene expression data were logarithmically transformed to yield normally distributed values, and are presented as $\log _{2}\left(2^{-\Delta C T}\right)$. For global transcriptome analysis, RNA was collected from TGF $\beta$ 1-treated organoids after $6,24,48$, and $72 \mathrm{~h}$, and from solvent-treated controls after 0 and $72 \mathrm{~h}$ of cultivation and paired-end sequenced on an Illumina HiSeq4000 at the Genome and Proteome Core Facility of the German Cancer Research Center, Heidelberg, Germany. FASTQ files were trimmed for sequencing adapters and low-quality reads with Trimmomatic (63). Reads were aligned to the Ensembl genome GRCM38 and reads per gene were quantified using STAR (64). For statistical analyses the R/Bioconductor package edgeR was used (65). We matched Ensembl IDs with EntrezIDs. If multiple Ensembl IDs matched to more than one Entrez ID, the one with the largest inter-quartile-range across samples was kept. Genes with less than one count per million in at least 3 samples were filtered out. Data were normalized with TMM (trimmed mean of M-values). Tagwise dispersion was calculated using edgeR. EdgeR was also used to compare data of the time series of TGF $\beta 1$ treatment to the $0 \mathrm{~h}$ and $72 \mathrm{~h}$ solvent controls. Differentially expressed genes (DEG) were defined by an adj. $p$-value $<0.01$ and a $\left|\log _{2}(\mathrm{FC})\right|>1$. Fisher's exact test for Gene Ontology biological processes (GO:BP) (66), ConsensusPathDB (67) and a selected group of EMT signatures $(41,43,40,44,42)$ was applied for functional enrichment analysis of DEGs. Terms were 
considered to be significantly regulated at an adj. $p$-value $<0.05$. All RNA-seq data were deposited in the Gene Expression Omnibus with the accession code GSE156553.

CMS classification. The R/Bioconductor package CMScaller was used to determine the CMS of a given sample (68). For organoid transcriptome data, mouse genes were first mapped to their human orthologs using the getHomoGenelDs function from the R/Bioconductor package GeneAnswers with the direct mapping method (69).

Analysis of colon cancer data. Colon cancer RNA-seq data were downloaded from TCGA firehose (https://gdac.broadinstitute.org). Following CMS classification, only CMS2 and CMS4 samples were further processed. For these, we matched Ensembl IDs with EntrezIDs and, if multiple Ensembl IDs were matched to more than one Entrez ID, the one with the largest inter-quartile-range across samples was kept. Genes with less than one count per million in at least 3 samples were filtered out. TMM normalization, tagwise dispersion, and statistical analysis using edgeR were performed similarly to described above. For comparison of CMS4 to CMS2 samples, DEGs were defined by an adj. $p$-value $<0.01$ and $\left|\log _{2}(F C)\right|>1$ as calculated with edgeR. DEGs were subjected to functional enrichment analysis using Fisher's exact test for the selected group of previously defined EMT signatures. A signature was considered to be significantly regulated if the adj. $p$-value $<0.05$.

Protein expression analysis. Organoids were incubated in Cell Recovery Solution and nuclear extracts were prepared by resuspending organoids in nuclear extraction buffer $A$ (10 mM HEPES/KOH pH 7.9, 0.1 mM EDTA, 10 mM KCl, 1x Complete ${ }^{\circledR}$ [Roche, 1697498], 1 mM DTT, 1x phosphatase inhibitor cocktails 2 and 3 [Sigma Aldrich, P5726/P0044]). After incubation on ice for $15 \mathrm{~min}, 0.5 \%$ (v/v) NP-40 was added, organoid suspensions were shortly vortexed, and nuclei were pelleted by centrifugation at $4^{\circ} \mathrm{C}$ and $16,100 \mathrm{~g}$ for 2 min. The cytosolic supernatant was collected, and the nuclear pellet was washed once with nuclear extraction buffer $A$. Thereafter, nuclei were resuspended in $20 \mathrm{mM} \mathrm{HEPES/KOH} \mathrm{pH}$ 
7.9, $400 \mathrm{mM} \mathrm{NaCl}, 1 \mathrm{mM}$ EDTA, 1x Complete ${ }^{\circledR}, 1 \mathrm{mM}$ DTT, 1x phosphatase inhibitor cocktails 2 and 3 , and incubated at $4^{\circ} \mathrm{C}$ for 30 min with constant shaking. Nuclear extracts were cleared by centrifugation at $4^{\circ} \mathrm{C}$ and $16,100 \mathrm{~g}$ for $10 \mathrm{~min}$. Protein concentrations were determined with the BioRad DC ${ }^{\mathrm{TM}}$ Protein Assay (BioRad, 500-0113). Depending on the protein yield in a given experiment, $25-40 \mu \mathrm{g}$ of protein was separated by SDS-PAGE and transferred to nitrocellulose for protein detection as described (70). Antibodies are listed in Supplementary table 7 .

Query of publicly available cancer genome data and ISMARA. Genetic alterations in components of the TGF $\beta$ pathway were determined in CRC samples (47) using cBioPortal $(71,72)$. TF motif activities were predicted by the web-based tool ISMARA (45). Motifs were collected and sorted according to their significance as determined by their z-values. Motif activity profiles for TFs related to different EMT states were extracted according to ref. (7).

Statistics and software. Data were analyzed and visualized using GraphPad Prism 5 (GraphPad Software) and RStudio (73). For time-resolved targeted gene expression studies, statistical analyses were done using a linear model allowing for simultaneous statistical testing for different time points. Normal distribution of the data was assessed with the car package (74). We applied the $\operatorname{Im}($ ) function and defined the solvent control as intercept to estimate effect sizes and calculate $p$-values based on t-statistics. Analyses were completed by Bonferroni correction for multiple comparisons with the same data. In experiments in which two populations were compared, statistical significance was assessed using the twotailed Mann-Whitney $U$ test with a confidence interval of $95 \%$. Box plots were generated with ggplot2 (75) and display the median with the lower and upper quartile. Whiskers show 1.5 times the interquartile range. Final figures were assembled using Canvas X 2017 (Canvas GFX, Inc.). 


\section{References}

1. Valastyan S, Weinberg RA. Tumor metastasis: molecular insights and evolving paradigms. Cell 2011; 147(2):275-92.

2. Friedl P, Locker J, Sahai E, Segall JE. Classifying collective cancer cell invasion. Nat Cell Biol 2012; 14(8):777-83.

3. Bronsert P, Enderle-Ammour K, Bader M, Timme S, Kuehs M, Csanadi A et al. Cancer cell invasion and EMT marker expression: a three-dimensional study of the human cancerhost interface. J Pathol 2014; 234(3):410-22.

4. Dongre A, Weinberg RA. New insights into the mechanisms of epithelial-mesenchymal transition and implications for cancer. Nat Rev Mol Cell Biol 2019; 20(2):69-84.

5. Lamouille S, Xu J, Derynck R. Molecular mechanisms of epithelial-mesenchymal transition. Nat Rev Mol Cell Biol 2014; 15(3):178-96.

6. Pastushenko I, Brisebarre A, Sifrim A, Fioramonti M, Revenco T, Boumahdi S et al. Identification of the tumour transition states occurring during EMT. Nature 2018; 556(7702):463-8.

7. Pastushenko I, Blanpain C. EMT Transition States during Tumor Progression and Metastasis. Trends Cell Biol 2019; 29(3):212-26.

8. Zhang J, Tian X-J, Zhang H, Teng Y, Li R, Bai F et al. TGF- $\beta$-induced epithelial-tomesenchymal transition proceeds through stepwise activation of multiple feedback loops. Sci Signal 2014; 7(345):ra91.

9. Puram SV, Tirosh I, Parikh AS, Patel AP, Yizhak K, Gillespie S et al. Single-Cell Transcriptomic Analysis of Primary and Metastatic Tumor Ecosystems in Head and Neck Cancer. Cell 2017; 171(7):1611-1624.e24.

10. Aiello NM, Maddipati R, Norgard RJ, Balli D, Li J, Yuan S et al. EMT Subtype Influences Epithelial Plasticity and Mode of Cell Migration. Dev Cell 2018; 45(6):681-695.e4. 
11. Kröger C, Afeyan A, Mraz J, Eaton EN, Reinhardt F, Khodor YL et al. Acquisition of a hybrid E/M state is essential for tumorigenicity of basal breast cancer cells. Proc Natl Acad Sci U S A 2019; 116(15):7353-62.

12. McFaline-Figueroa JL, Hill AJ, Qiu X, Jackson D, Shendure J, Trapnell C. A pooled single-cell genetic screen identifies regulatory checkpoints in the continuum of the epithelial-to-mesenchymal transition. Nat Genet 2019; 51(9):1389-98.

13. Boutin AT, Liao W-T, Wang M, Hwang SS, Karpinets TV, Cheung H et al. Oncogenic Kras drives invasion and maintains metastases in colorectal cancer. Genes Dev 2017; 31(4):370-82.

14. Roper J, Tammela T, Cetinbas NM, Akkad A, Roghanian A, Rickelt S et al. In vivo genome editing and organoid transplantation models of colorectal cancer and metastasis. Nat Biotechnol 2017; 35(6):569-76.

15. O'Rourke KP, Loizou E, Livshits G, Schatoff EM, Baslan T, Manchado E et al. Transplantation of engineered organoids enables rapid generation of metastatic mouse models of colorectal cancer. Nat Biotechnol 2017; 35(6):577-82.

16. Fumagalli A, Oost KC, Kester L, Morgner J, Bornes L, Bruens L et al. Plasticity of Lgr5Negative Cancer Cells Drives Metastasis in Colorectal Cancer. Cell Stem Cell 2020; 26(4):569-578.e7.

17. Drost J, van Jaarsveld RH, Ponsioen B, Zimberlin C, van Boxtel R, Buijs A et al. Sequential cancer mutations in cultured human intestinal stem cells. Nature 2015; $521(7550): 43-7$.

18. Matano M, Date S, Shimokawa M, Takano A, Fujii M, Ohta Y et al. Modeling colorectal cancer using CRISPR-Cas9-mediated engineering of human intestinal organoids. Nat Med 2015; 21(3):256-62.

19. Li X, Nadauld L, Ootani A, Corney DC, Pai RK, Gevaert O et al. Oncogenic transformation of diverse gastrointestinal tissues in primary organoid culture. Nat Med 2014; 20(7):769-77. 
20. Sakai E, Nakayama M, Oshima H, Kouyama Y, Niida A, Fujii S et al. Combined Mutation of Apc, Kras, and Tgfbr2 Effectively Drives Metastasis of Intestinal Cancer. Cancer Res 2018; 78(5):1334-46.

21. Fumagalli A, Drost J, Suijkerbuijk SJE, van Boxtel R, Ligt $J$ de, Offerhaus GJ et al. Genetic dissection of colorectal cancer progression by orthotopic transplantation of engineered cancer organoids. Proc Natl Acad Sci U S A 2017; 114(12):E2357-E2364.

22. Calon A, Espinet E, Palomo-Ponce S, Tauriello DVF, Iglesias M, Céspedes MV et al. Dependency of colorectal cancer on a TGF- $\beta$-driven program in stromal cells for metastasis initiation. Cancer Cell 2012; 22(5):571-84.

23. Tauriello DVF, Palomo-Ponce S, Stork D, Berenguer-Llergo A, Badia-Ramentol J, Iglesias $M$ et al. TGF $\beta$ drives immune evasion in genetically reconstituted colon cancer metastasis. Nature 2018; 554(7693):538-43.

24. Cancer Genome Atlas Network. Comprehensive molecular characterization of human colon and rectal cancer. Nature 2012; 487(7407):330.

25. Guinney J, Dienstmann R, Wang X, Reyniès A de, Schlicker A, Soneson C et al. The consensus molecular subtypes of colorectal cancer. Nat Med 2015; 21(11):1350-6.

26. Fessler E, Drost J, van Hooff SR, Linnekamp JF, Wang $X$, Jansen $M$ et al. TGF $\beta$ signaling directs serrated adenomas to the mesenchymal colorectal cancer subtype. EMBO Mol Med 2016; 8(7):745-60.

27. Giampieri S, Manning C, Hooper S, Jones L, Hill CS, Sahai E. Localized and reversible TGFbeta signalling switches breast cancer cells from cohesive to single cell motility. Nat Cell Biol 2009; 11(11):1287-96.

28. Dekkers JF, Wiegerinck CL, Jonge HR de, Bronsveld I, Janssens HM, Winter-de Groot $\mathrm{KM}$ de et al. A functional CFTR assay using primary cystic fibrosis intestinal organoids. Nat Med 2013; 19(7):939-45.

29. Nissen NI, Karsdal M, Willumsen N. Collagens and Cancer associated fibroblasts in the reactive stroma and its relation to Cancer biology. J Exp Clin Cancer Res 2019; $38(1): 115$. 
30. Wiener Z, Band AM, Kallio P, Högström J, Hyvönen V, Kaijalainen S et al. Oncogenic mutations in intestinal adenomas regulate Bim-mediated apoptosis induced by TGF- $\beta$. Proc Natl Acad Sci U S A 2014; 111(21):E2229-36.

31. Singh P, Carraher C, Schwarzbauer JE. Assembly of fibronectin extracellular matrix. Annu Rev Cell Dev Biol 2010; 26:397-419.

32. Danen EHJ, Sonneveld P, Brakebusch C, Fassler R, Sonnenberg A. The fibronectinbinding integrins alpha5beta1 and alphavbeta3 differentially modulate RhoA-GTP loading, organization of cell matrix adhesions, and fibronectin fibrillogenesis. J Cell Biol 2002; 159(6):1071-86.

33. Bays JL, DeMali KA. Vinculin in cell-cell and cell-matrix adhesions. Cell Mol Life Sci 2017; 74(16):2999-3009.

34. Maschler S, Wirl G, Spring H, Bredow DV, Sordat I, Beug H et al. Tumor cell invasiveness correlates with changes in integrin expression and localization. Oncogene 2005; 24(12):2032-41.

35. Mise N, Savai R, Yu H, Schwarz J, Kaminski N, Eickelberg O. Zyxin is a transforming growth factor- $\beta$ (TGF- $\beta$ )/Smad3 target gene that regulates lung cancer cell motility via integrin $\alpha 5 \beta 1$. J Biol Chem 2012; 287(37):31393-405.

36. Kuonen F, Surbeck I, Sarin KY, Dontenwill M, Rüegg C, Gilliet M et al. TGF $\beta$, Fibronectin and Integrin a5ß1 Promote Invasion in Basal Cell Carcinoma. J Invest Dermatol 2018; 138(11):2432-42.

37. Morén A, Imamura T, Miyazono K, Heldin C-H, Moustakas A. Degradation of the tumor suppressor Smad4 by WW and HECT domain ubiquitin ligases. J Biol Chem 2005; 280(23):22115-23.

38. Siegel PM, Shu W, Cardiff RD, Muller WJ, Massagué J. Transforming growth factor beta signaling impairs Neu-induced mammary tumorigenesis while promoting pulmonary metastasis. Proc Natl Acad Sci U S A 2003; 100(14):8430-5.

39. Vega S, Morales AV, Ocaña OH, Valdés F, Fabregat I, Nieto MA. Snail blocks the cell cycle and confers resistance to cell death. Genes Dev 2004; 18(10):1131-43. 
40. Jechlinger M, Grunert S, Tamir IH, Janda E, Lüdemann S, Waerner T et al. Expression profiling of epithelial plasticity in tumor progression. Oncogene 2003; 22(46):7155-69.

41. Gotzmann J, Fischer ANM, Zojer M, Mikula M, Proell V, Huber H et al. A crucial function of PDGF in TGF-beta-mediated cancer progression of hepatocytes. Oncogene 2006; 25(22):3170-85.

42. Taube JH, Herschkowitz JI, Komurov K, Zhou AY, Gupta S, Yang J et al. Core epithelialto-mesenchymal transition interactome gene-expression signature is associated with claudin-low and metaplastic breast cancer subtypes. Proc Natl Acad Sci U S A 2010; 107(35):15449-54.

43. Gröger CJ, Grubinger M, Waldhör T, Vierlinger K, Mikulits W. Meta-analysis of gene expression signatures defining the epithelial to mesenchymal transition during cancer progression. PLoS ONE 2012; 7(12):e51136.

44. Mak MP, Tong P, Diao L, Cardnell RJ, Gibbons DL, William WN et al. A Patient-Derived, Pan-Cancer EMT Signature Identifies Global Molecular Alterations and Immune Target Enrichment Following Epithelial-to-Mesenchymal Transition. Clin Cancer Res 2016; 22(3):609-20.

45. Balwierz PJ, Pachkov M, Arnold P, Gruber AJ, Zavolan M, van Nimwegen E. ISMARA: automated modeling of genomic signals as a democracy of regulatory motifs. Genome Res 2014; 24(5):869-84.

46. Stemmler MP, Eccles RL, Brabletz S, Brabletz T. Non-redundant functions of EMT transcription factors. Nat Cell Biol 2019; 21(1):102-12.

47. Yaeger R, Chatila WK, Lipsyc MD, Hechtman JF, Cercek A, Sanchez-Vega F et al. Clinical Sequencing Defines the Genomic Landscape of Metastatic Colorectal Cancer. Cancer Cell 2018; 33(1):125-136.e3.

48. Joyce JA, Pollard JW. Microenvironmental regulation of metastasis. Nat Rev Cancer 2009; 9(4):239-52.

49. Tauriello DVF, Calon A, Lonardo E, Batlle E. Determinants of metastatic competency in colorectal cancer. Mol Oncol 2017; 11(1):97-119. 
50. Lu P, Weaver VM, Werb Z. The extracellular matrix: a dynamic niche in cancer progression. J Cell Biol 2012; 196(4):395-406.

51. Pape J, Magdeldin T, Stamati K, Nyga A, Loizidou M, Emberton M et al. Cancerassociated fibroblasts mediate cancer progression and remodel the tumouroid stroma. $\mathrm{Br}$ J Cancer 2020.

52. Zheng X, Carstens JL, Kim J, Scheible M, Kaye J, Sugimoto H et al. Epithelial-tomesenchymal transition is dispensable for metastasis but induces chemoresistance in pancreatic cancer. Nature 2015; 527(7579):525-30.

53. el Marjou F, Janssen K-P, Chang BH-J, Li M, Hindie V, Chan L et al. Tissue-specific and inducible Cre-mediated recombination in the gut epithelium. Genesis 2004; 39(3):18693.

54. Shibata H, Toyama K, Shioya H, Ito M, Hirota M, Hasegawa S et al. Rapid colorectal adenoma formation initiated by conditional targeting of the Apc gene. Science 1997; 278(5335):120-3.

55. Tuveson DA, Shaw AT, Willis NA, Silver DP, Jackson EL, Chang S et al. Endogenous oncogenic K-rasG12D stimulates proliferation and widespread neoplastic and developmental defects. Cancer Cell 2004; 5(4):375-87.

56. Olive KP, Tuveson DA, Ruhe ZC, Yin B, Willis NA, Bronson RT et al. Mutant p53 gain of function in two mouse models of Li-Fraumeni syndrome. Cell 2004; 119(6):847-60.

57. Wenzel J, Rose K, Haghighi EB, Lamprecht C, Rauen G, Freihen V et al. Loss of the nuclear Wnt pathway effector TCF7L2 promotes migration and invasion of human colorectal cancer cells. Oncogene 2020; 39(19):3893-909.

58. Rönsch K, Jägle S, Rose K, Seidl M, Baumgartner F, Freihen V et al. SNAIL1 combines competitive displacement of ASCL2 and epigenetic mechanisms to rapidly silence the EPHB3 tumor suppressor in colorectal cancer. Mol Oncol 2015; 9(2):335-54.

59. Schnappauf O, Beyes S, Dertmann A, Freihen V, Frey $P$, Jägle $S$ et al. Enhancer decommissioning by Snail1-induced competitive displacement of TCF7L2 and down- 
regulation of transcriptional activators results in EPHB2 silencing. Biochim Biophys Acta 2016; 1859(11):1353-67.

60. Stemmer M, Thumberger T, Del Sol Keyer M, Wittbrodt J, Mateo JL. CCTop: An Intuitive, Flexible and Reliable CRISPR/Cas9 Target Prediction Tool. PLoS ONE 2015; 10(4):e0124633.

61. Nyström A, Thriene K, Mittapalli V, Kern JS, Kiritsi D, Dengjel J et al. Losartan ameliorates dystrophic epidermolysis bullosa and uncovers new disease mechanisms. EMBO Mol Med 2015; 7(9):1211-28.

62. Freihen V, Rönsch K, Mastroianni J, Frey P, Rose K, Boerries M et al. SNAIL1 employs $\beta$-Catenin-LEF1 complexes to control colorectal cancer cell invasion and proliferation. Int J Cancer 2020; 146(8):2229-42.

63. Bolger AM, Lohse M, Usadel B. Trimmomatic: a flexible trimmer for Illumina sequence data. Bioinformatics 2014; 30(15):2114-20.

64. Dobin A, Davis CA, Schlesinger F, Drenkow J, Zaleski C, Jha S et al. STAR: ultrafast universal RNA-seq aligner. Bioinformatics 2013; 29(1):15-21.

65. Robinson MD, McCarthy DJ, Smyth GK. edgeR: a Bioconductor package for differential expression analysis of digital gene expression data. Bioinformatics 2010; 26(1):139-40.

66. Subramanian A, Tamayo P, Mootha VK, Mukherjee S, Ebert BL, Gillette MA et al. Gene set enrichment analysis: a knowledge-based approach for interpreting genome-wide expression profiles. Proc Natl Acad Sci U S A 2005; 102(43):15545-50.

67. Kamburov A, Wierling C, Lehrach H, Herwig R. ConsensusPathDB--a database for integrating human functional interaction networks. Nucleic Acids Res 2009; 37(Database issue):D623-8.

68. Eide PW, Bruun J, Lothe RA, Sveen A. CMScaller: an R package for consensus molecular subtyping of colorectal cancer pre-clinical models. Sci Rep 2017; 7(1):16618.

69. Feng G, Du P, Krett NL, Tessel M, Rosen S, Kibbe WA et al. A collection of bioconductor methods to visualize gene-list annotations. BMC research notes 2010; 3:10. 
70. Weise A, Bruser K, Elfert S, Wallmen B, Wittel Y, Wöhrle S et al. Alternative splicing of Tcf7l2 transcripts generates protein variants with differential promoter-binding and transcriptional activation properties at Wnt/beta-catenin targets. Nucleic Acids Res 2010; 38(6):1964-81.

71. Cerami E, Gao J, Dogrusoz U, Gross BE, Sumer SO, Aksoy BA et al. The cBio cancer genomics portal: an open platform for exploring multidimensional cancer genomics data. Cancer Discov 2012; 2(5):401-4.

72. Gao J, Aksoy BA, Dogrusoz U, Dresdner G, Gross B, Sumer SO et al. Integrative analysis of complex cancer genomics and clinical profiles using the cBioPortal. Sci Signal 2013; 6(269):pl1.

73. RStudio Team. RStudio: Integrated Development for R.; 2015.

74. Fox J, Weisberg S. An R companion to applied regression. Third edition. Thousand Oaks, California: SAGE; 2019.

75. Wickham H. ggplot2: Elegant graphics for data analysis. Cham: Springer; 2016. (Use R!). 
a

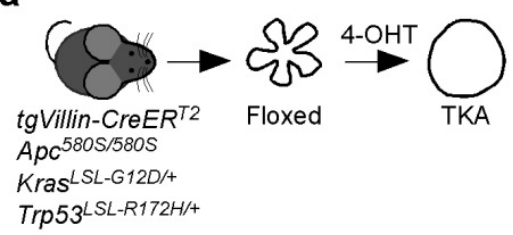

C
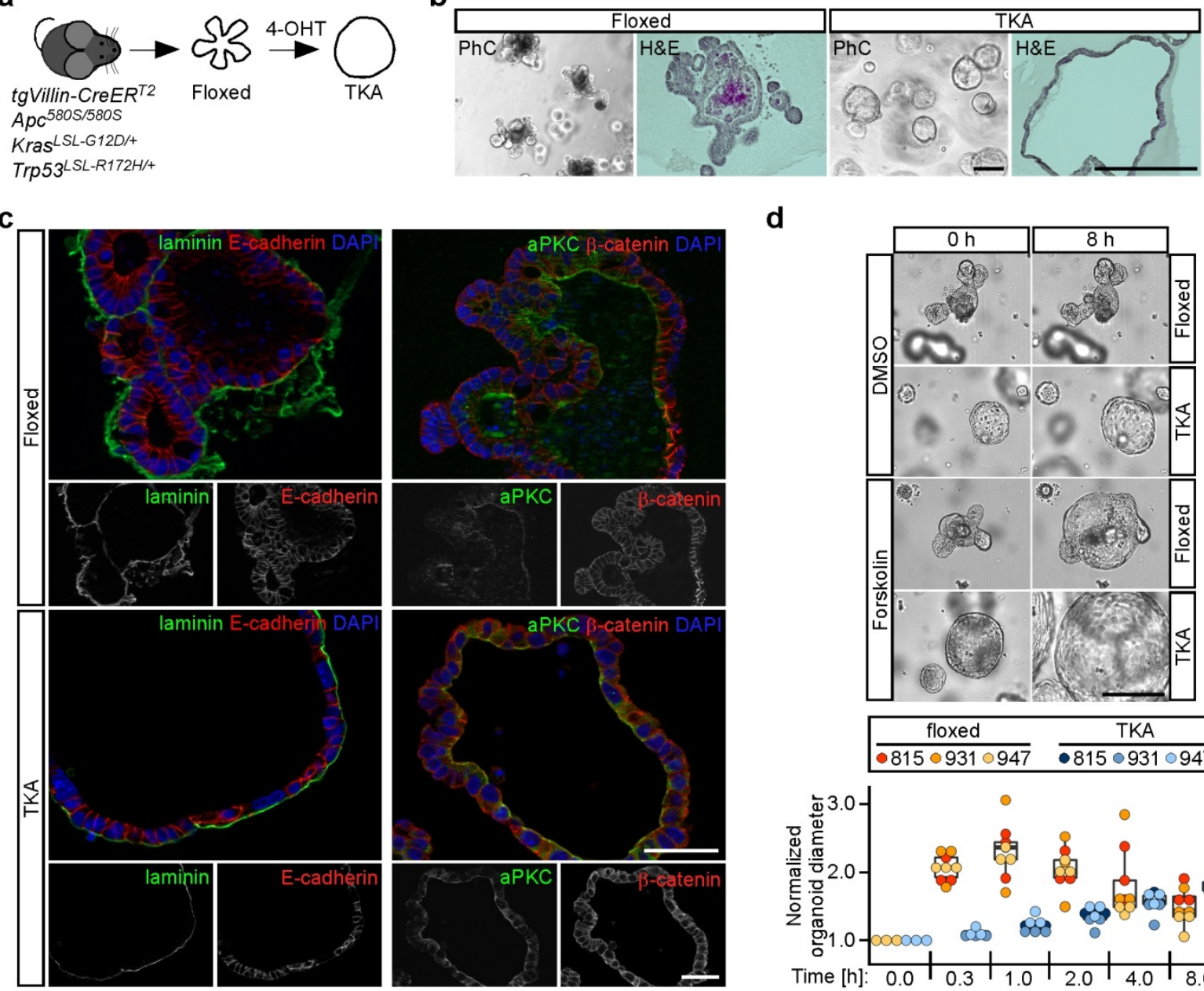

d
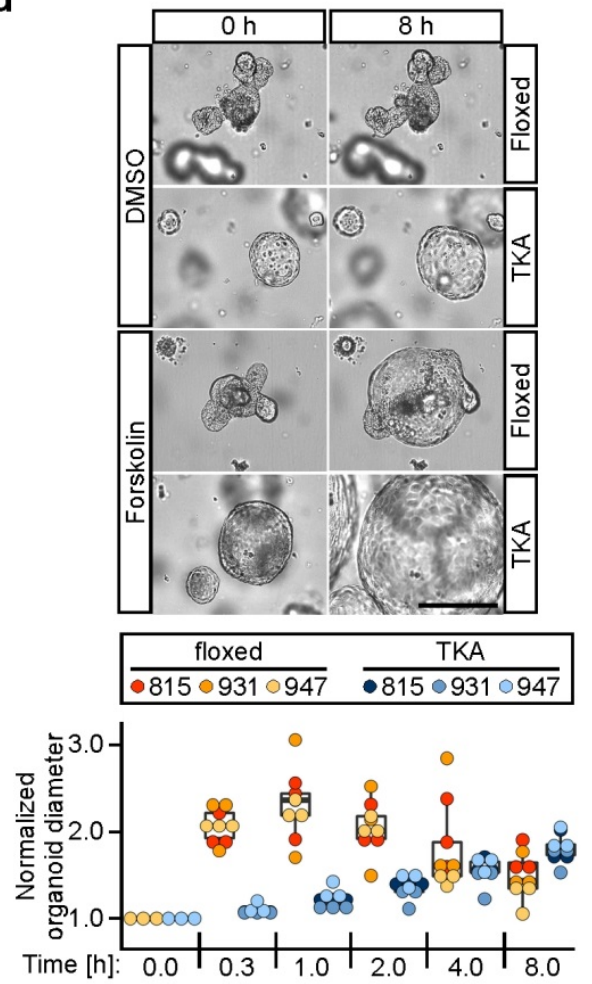

e

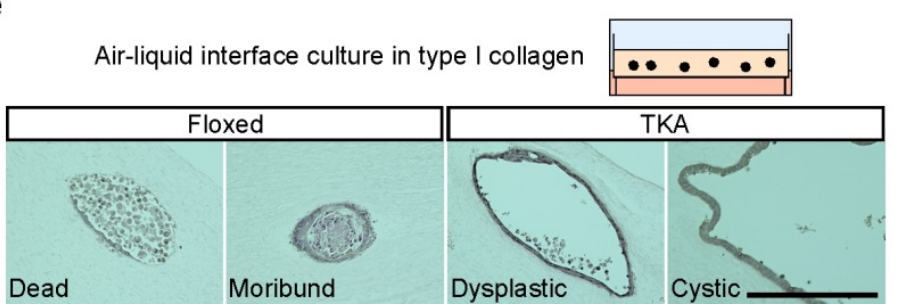

Fig. 1: Oncogenically transformed intestinal organoids display no cell-intrinsic

invasiveness in vitro. a, Strategy for generating oncogenically transformed small intestinal and colonic organoids from genetically engineered mice carrying a Villin-CreE $R^{T 2}$ transgene, two floxed Apc alleles, as well as heterozygous $K r a s^{L S L-G 12 D /+}$ and $\operatorname{Trp} 53^{L S L-R 172 H /+}$ loci. Floxed stop cassettes (LSL) prevent expression of the mutant Kras and Trp53 alleles. For recombination, organoids were treated with 4-hydroxy-tamoxifen (4-OHT), yielding TKA organoids. b, Whole mounts and hematoxylin/eosin (H\&E) stained sections of floxed and TKA organoids (line 815 ) cultured in $7 \mathrm{mg} / \mathrm{ml}$ Matrigel and visualized by phase contrast 
(PhC) or bright field microscopy. Scale bars: $200 \mu \mathrm{m}$. c, Representative images of immunofluorescence staining of pan-laminin, E-cadherin, atypical protein kinase C (aPKC), and $\beta$-catenin in sections of floxed and TKA organoids (line 815 ) cultured in $7 \mathrm{mg} / \mathrm{ml}$ Matrigel. Nuclei were stained by DAPI; $n>3$. Scale bars: $50 \mu \mathrm{m}$. d, Top: Microscopy of floxed and TKA organoids (line 815$)$ in $7 \mathrm{mg} / \mathrm{ml}$ Matrigel at the beginning $(0 \mathrm{~h})$ and the end $(8 \mathrm{~h})$ of forskolin and DMSO treatment. Scale bar: $200 \mu \mathrm{m}$. Bottom: Quantification of forskolininduced organoid swelling. TKA organoids were exposed to DMSO or forskolin for the indicated time periods. Normalized changes in organoid diameter were calculated by first computing at each time point and for each organoid under consideration the increase in diameter relative to the corresponding value at $\mathrm{t}=0 \mathrm{~h}$, followed by normalization of forskolininduced relative changes in diameter to those of DMSO-treated control samples. At least five organoids treated with DMSO or forskolin were analyzed per biological replicate and organoid line. e, Left: set-up for cultivating organoids in type I collagen at an air-liquidinterface and representative H\&E stainings of organoid displaying different histological features (line 815). Scale bar: $200 \mu \mathrm{m}$. Right: Quantification of organoids following histological classification (morib.: moribund; dyspl.: dysplastic). Quantitative experiments in (d and e) were performed with three floxed/TKA organoid lines (815: $n=3 ; 931: n=3 ; 947$ : $\mathrm{n}=3$ ). Dots represent results of independent biological replicates and dot color identifies the organoid lines. 
a

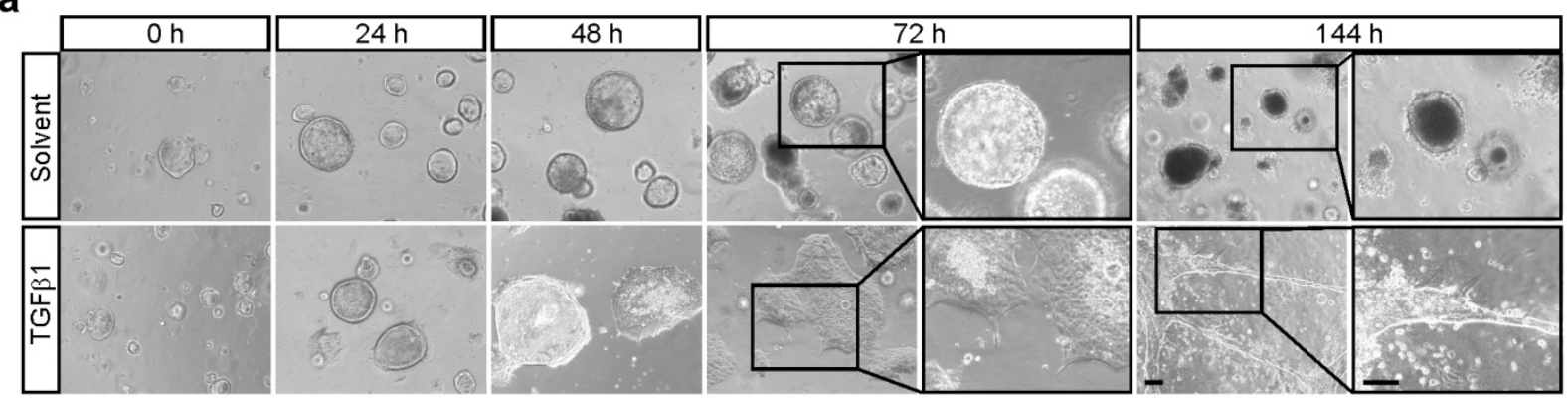

b

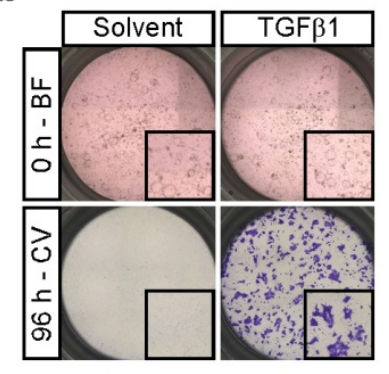

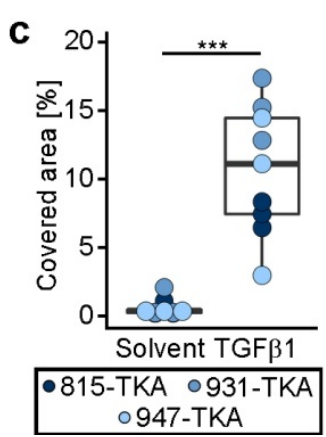

d

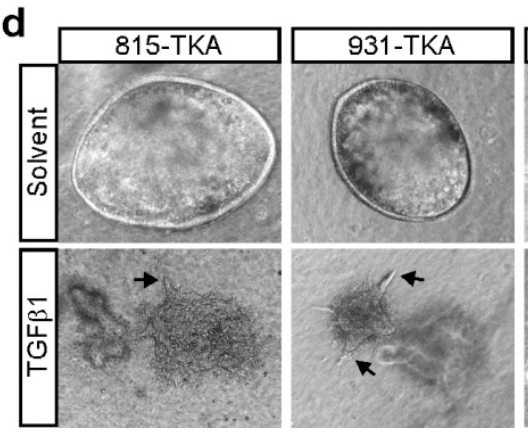

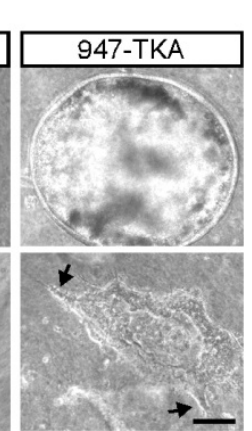

Fig. 2: TGF $\beta 1$ triggers collective invasion of TKA organoids. a, Morphological appearance of TKA organoids (line 815) cultured in $3 \mathrm{mg} / \mathrm{ml}$ Matrigel and treated with solvent or TGF $\beta 1$ for the indicated time periods. Boxed areas are shown at higher magnification on the right. Similar TGF $\beta 1$-induced morphological changes were observed with TKA organoid lines from five different founder mice. Scale bars: $100 \mu \mathrm{m}$. b. Boyden chamber invasion assays with TKA organoids (line 931) seeded in $3 \mathrm{mg} / \mathrm{ml}$ Matrigel. Top: bright field (BF) images taken at $0 \mathrm{~h}$ of TGF $\beta 1$ and solvent treatment. Inserts show magnified views of the upper chambers. Bottom: crystal violet (CV) staining of invaded cells after $96 \mathrm{~h}$ of treatment. Inserts show magnified views of the bottom faces of the Boyden chambers. c, Quantification of invasion experiments as shown in (b) performed with TKA organoid lines $815(n=3), 931$ $(n=3)$, and $947(n=3)$. Each dot represents the result of a single invasion assay while dot color identifies the organoid lines. ${ }^{* * *} p=0.0004$; Mann-Whitney $U$ test. d, Phase contrast microscopy of TKA organoids cultured in type I collagen in presence of solvent or TGF $\beta 1$. Arrows point at sites of collective invasion. Independent experiments were performed in three different organoid lines (815: $n=3 ; 931: n=3 ; 947: n=3)$. Scale bar: $100 \mu m$. 
a
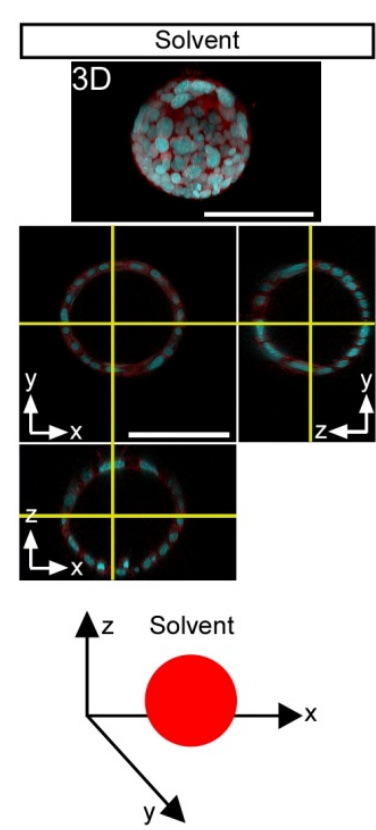
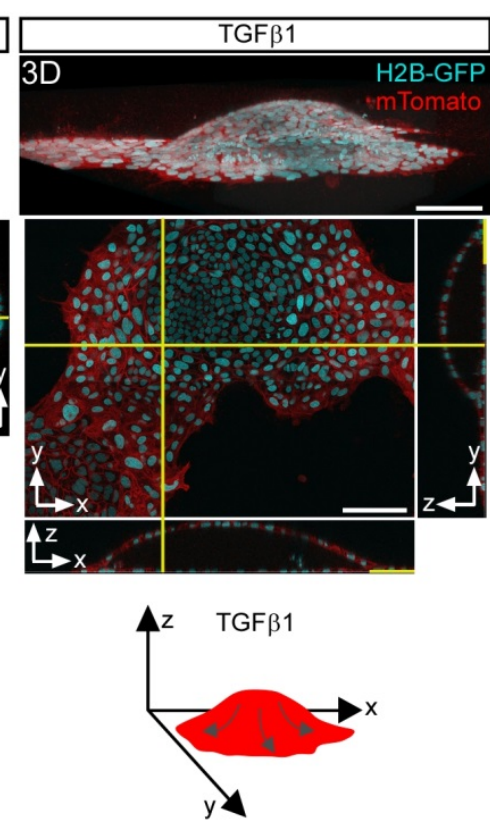
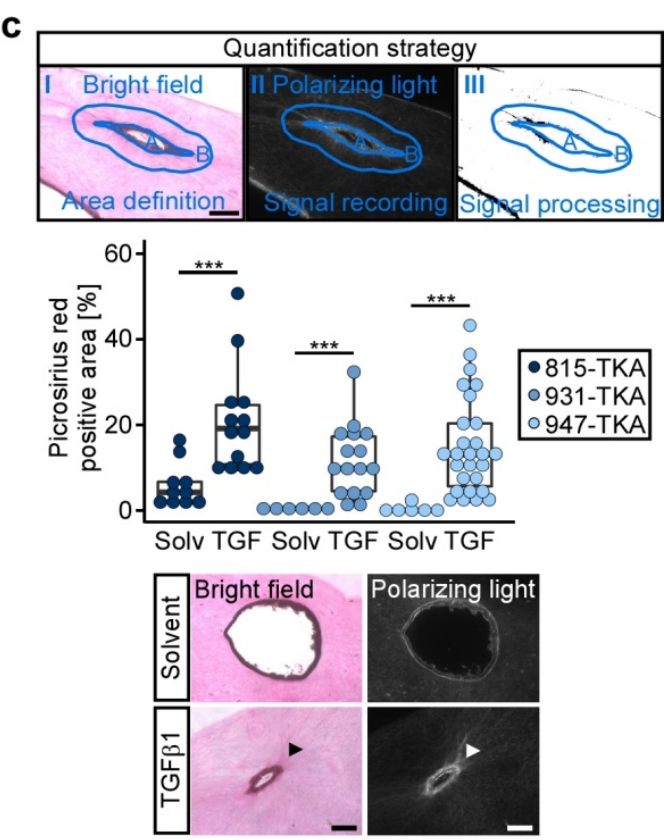

b

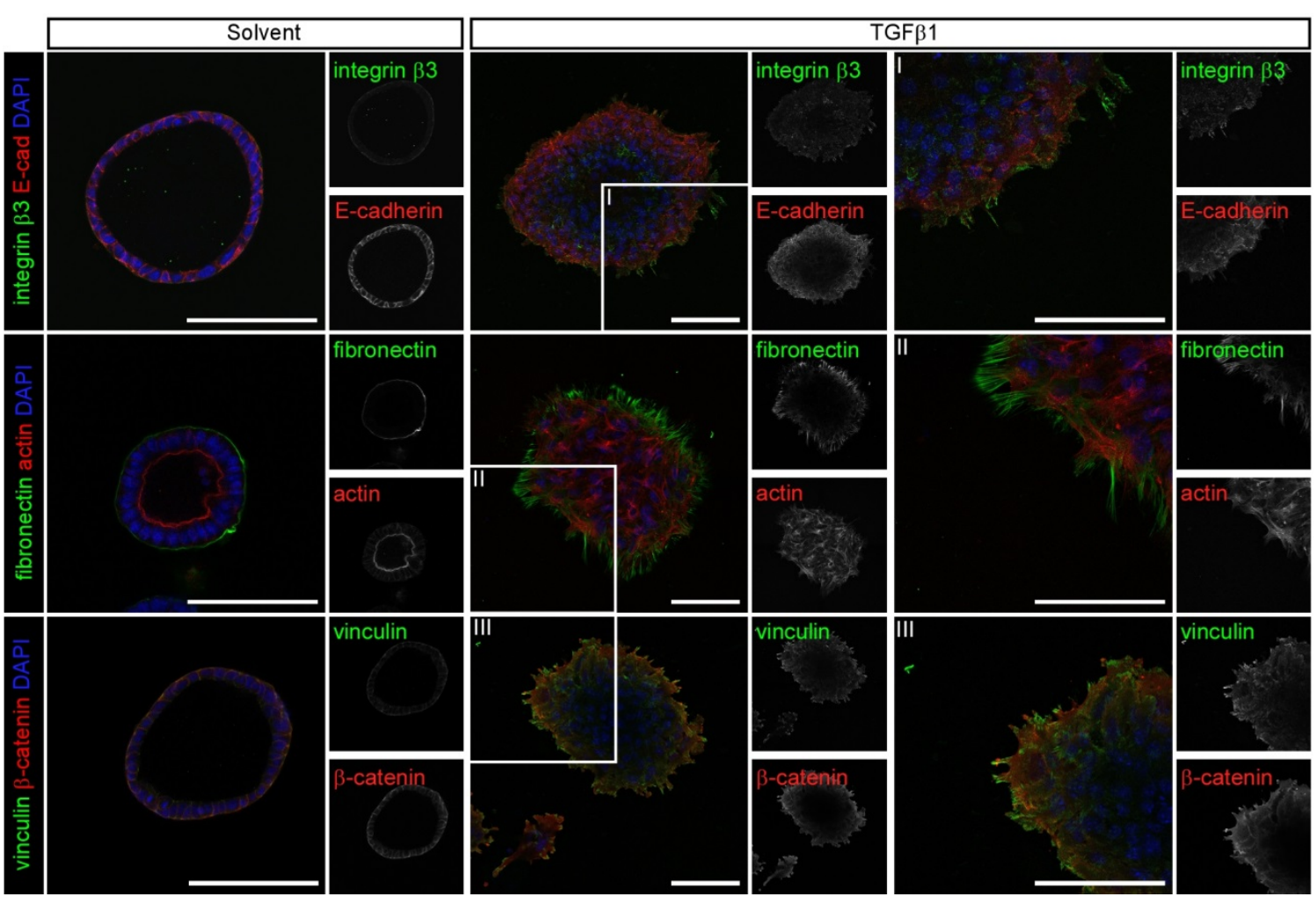

Fig. 3: TGF $\beta$ 1-stimulated TKA organoids interact with and remodel the ECM. a, TKA organoids (line 815) expressing nuclear H2B-GFP and membrane-bound mTomato were seeded in $3 \mathrm{mg} / \mathrm{ml}$ Matrigel, treated with solvent or TGF $\beta 1$ for $48 \mathrm{~h}$, and subjected to live imaging using confocal microscopy. Displayed are 3D reconstructions and orthogonal views of organoid cross-sections (yellow lines: positions of the cross-sections along the $x-, y-$ and 
z-axes). Scale bars: $100 \mu \mathrm{m}(\mathrm{n}=3)$. Bottom: schematic representations of TKA organoid morphologies. Arrows: proposed streaming of cells. b, Whole mount immunofluorescence staining and confocal microscopy of TKA organoids (line 815 ) seeded in $3 \mathrm{mg} / \mathrm{ml}$ Matrigel and treated with solvent or TGF $\beta 1$ for $72 \mathrm{~h}$. Organoids were stained with antibodies against the indicated antigens. Actin was visualized by phalloidin staining. Nuclei were labeled using DAPI. Boxed areas are shown at higher magnification on the right. Pictures are representative for results obtained with three TKA organoid lines (815: $n=1 ; 931: n=1 ; 947$ : $\mathrm{n}=1$ ). Scale bars: $100 \mu \mathrm{m}$. c, Top: strategy for quantification of picrosirius red staining: (I) organoids were visualized by bright field illumination and the outline of the organoids $(A)$ and a surrounding area (B) with an approximate width of $65 \mu \mathrm{m}$ were marked. (II) Collagen was stained by picrosirius red and larger or parallel collagen bundles were visualized by polarizing light and signal intensities across the entire image were recorded. (III) For the final quantifications, only signals within area B and exceeding a defined threshold were considered. To allow comparison of organoids with different sizes, signal intensities were normalized to the size of area B. Middle: quantifications of picrosirius red-stained TKA organoids cultured in type I collagen and treated with solvent (solv) or TGF $\beta 1$ (TGF) for $96 \mathrm{~h}$. Colored dots represent individual measurements from at least two independent experiments. Mann-Whitney $U$ test. TKA organoid line $815:{ }^{* * *} p=0.0007$ (solvent: $n=10$, TGF $\beta 1: n=13$ ); TKA organoid line 931: ${ }^{* *} p=0.0005$ (solvent: $n=6$, TGF $\beta 1: n=16$ ); TKA organoid line 947: ${ }^{* * *} p=0.0002$ (solvent: $\mathrm{n}=6$, TGF $\beta 1: \mathrm{n}=27$ ). Scale bar: $100 \mu \mathrm{m}$. Bottom: exemplary pictures of TKA organoids (line 815) treated with solvent or TGF $\beta 1$. 
a
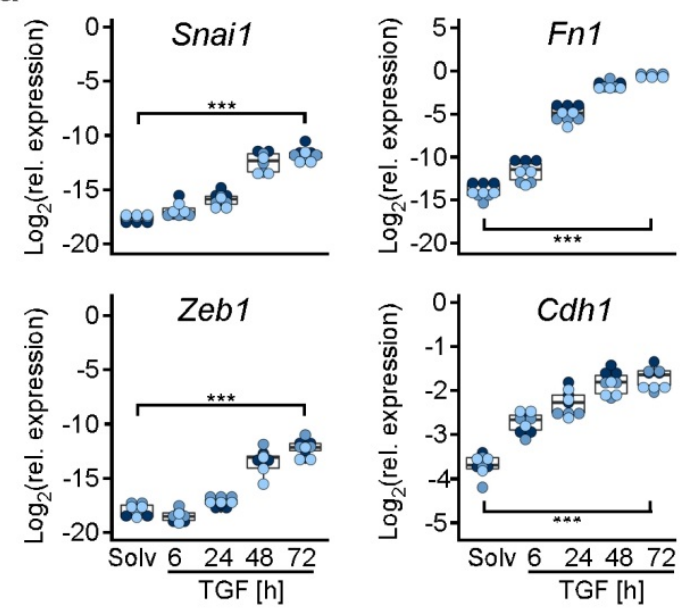

b

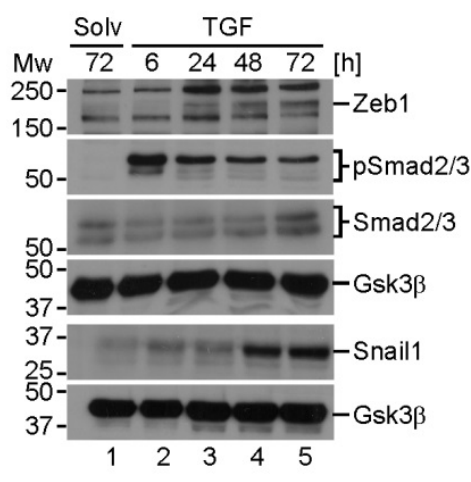

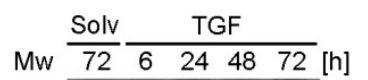

$150-1-$-integrin $\alpha 5$

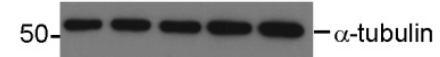

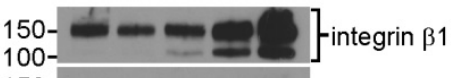

$150-=---$-Ephb3

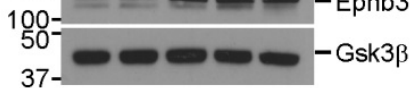

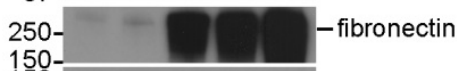

$150-$

$100--\infty-E-c a d$

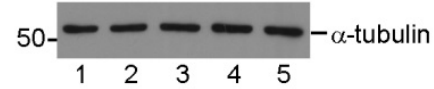
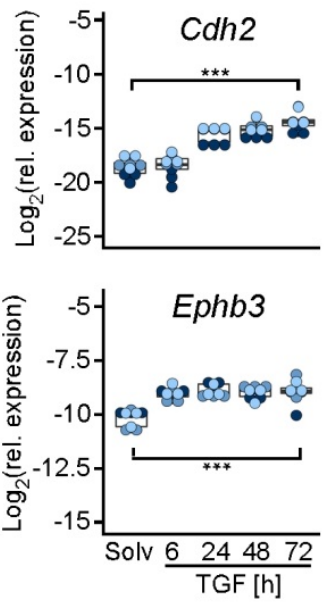

C

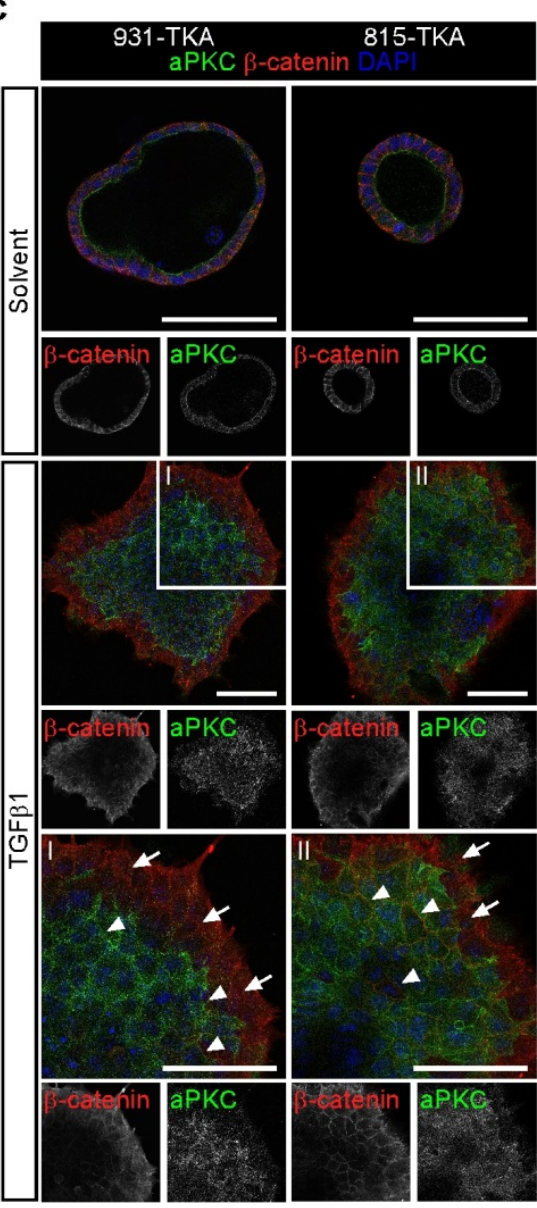

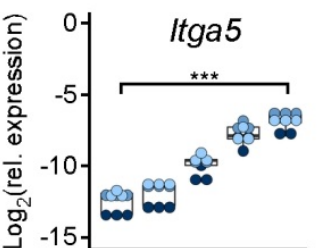

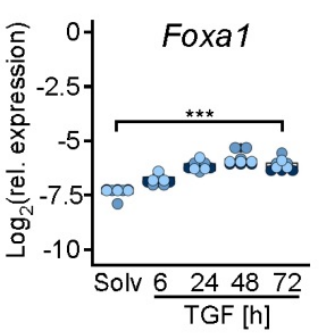

-815-TKA

-931-TKA 947-TKA
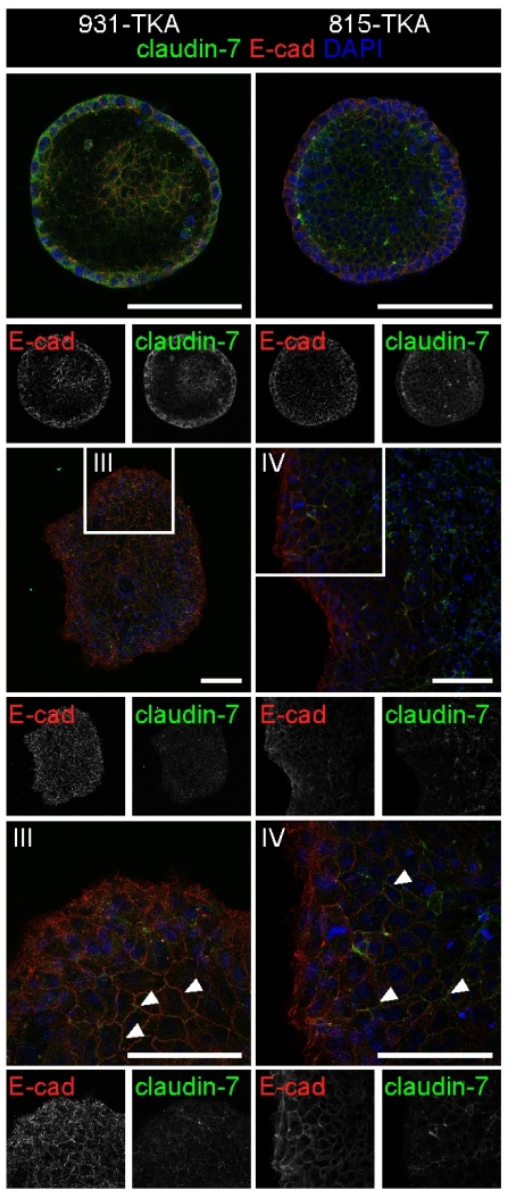

Fig. 4: TGF $\beta 1$ induces a partial EMT in TKA organoids. a, Time-resolved gene expression analyses of TKA organoids seeded in $3 \mathrm{mg} / \mathrm{ml}$ Matrigel and treated with solvent (solv) or TGF $\beta 1$ (TGF) for the indicated time periods. RNA levels of EMT-TFs and EMT-associated genes were quantified by qRT-PCR and normalized to transcript levels of Eef1a1. Each dot represents the result of a single measurement while dot color identifies the organoid lines. 
Three independent biological replicates were performed for each organoid line (815: $n=3$; 931: $n=3 ; 947: n=3) .{ }^{* * *} p<0.001$; statistical significance was analyzed using a linear regression model combined with Bonferroni correction for multiple comparisons. Exact $p$ values are provided in Supplementary table 1. b, Western blot analyses of phospho-Smad2/3 (pSmad2/3), total Smad2/3, EMT-TFs, and EMT-associated genes in TKA organoids (line 815) treated as in (a). Gsk3 $\beta$ and $\alpha$-tubulin were included as loading control. Molecular weights of size standards are given in kDa. Results are representative for experiments performed with three TKA organoid lines (815: $n=2 ; 931: n=2 ; 947: n=2$ ); E-cad: E-cadherin. c, Whole mount immunofluorescence staining and confocal microscopy of TKA organoid lines 815 and 931 seeded in $3 \mathrm{mg} / \mathrm{ml}$ Matrigel and treated with solvent or TGFß1 for $72 \mathrm{~h}$. Organoids were stained for E-cadherin, claudin-7, $\beta$-catenin, and atypical protein kinase C (aPKC). Arrowheads indicate membranous E-cadherin, claudin-7, and $\beta$-catenin in the central part of the TGF $\beta 1$-treated organoid. Arrows highlight reduced staining of aPKC in the peripheral region of TGF $\beta 1$-stimulated organoids. Boxed areas are shown at higher magnification below. Reduced staining intensities in some central regions of organoids might be caused by technical issues related to whole mount staining and confocal microscopy of organoids. Similar results were obtained with three different organoid lines $(815: n=1,931$ : $n=1,947: n=1)$. Scale bars: $100 \mu m$. 
a

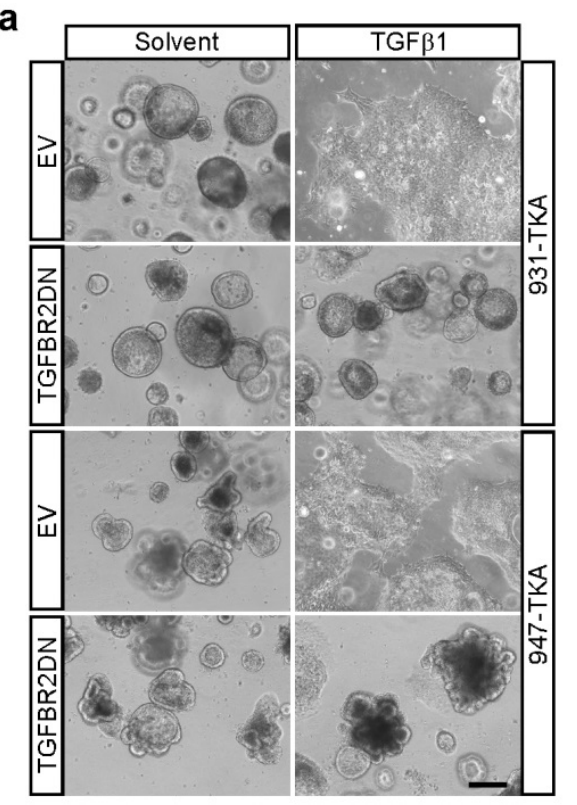

b
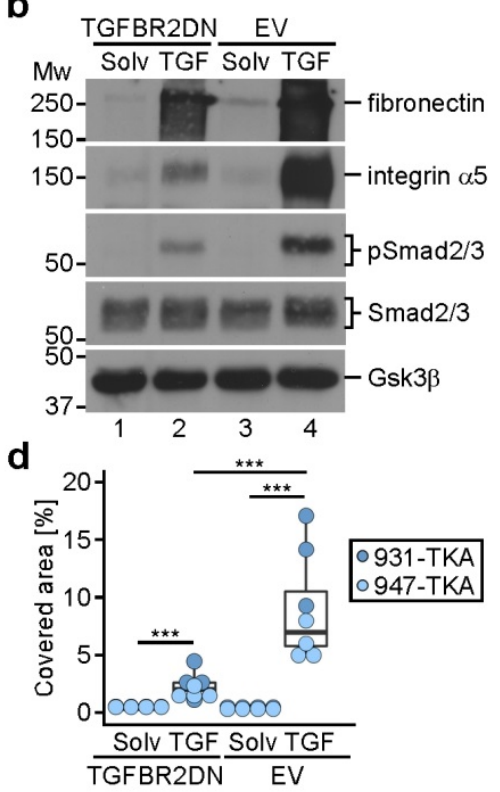

C

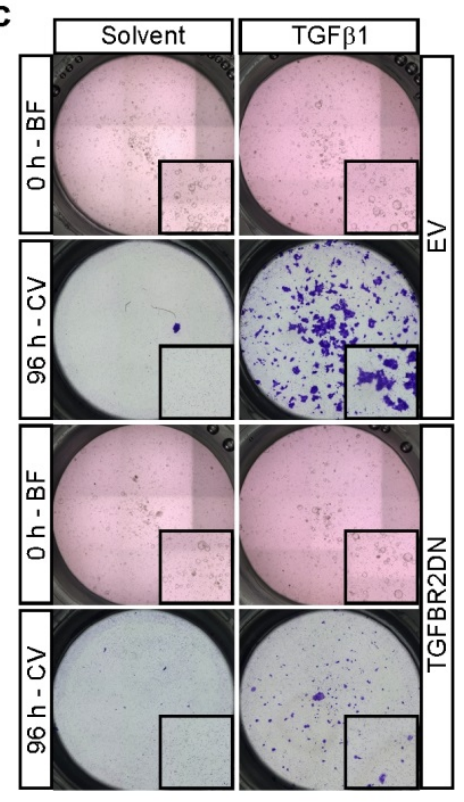

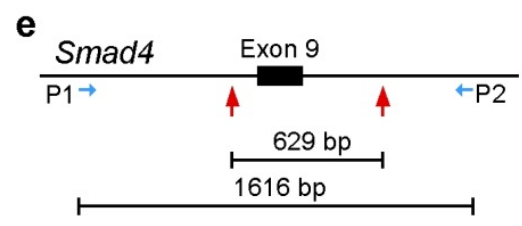

h
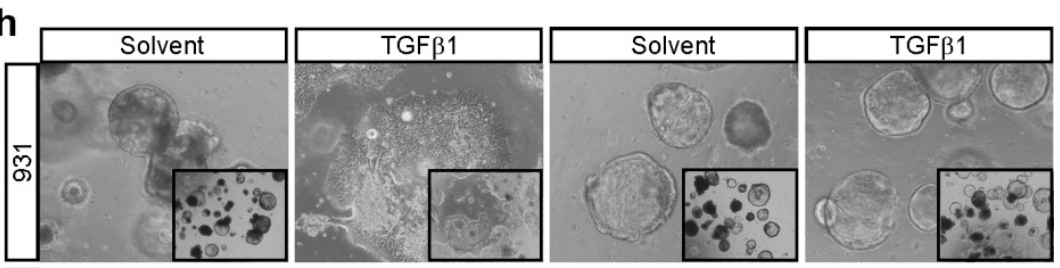

f
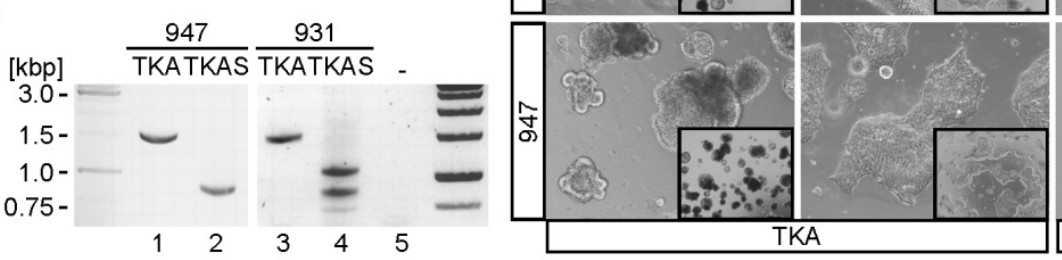

g

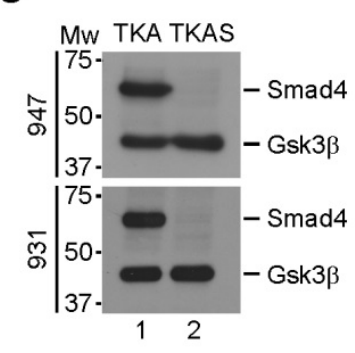

i

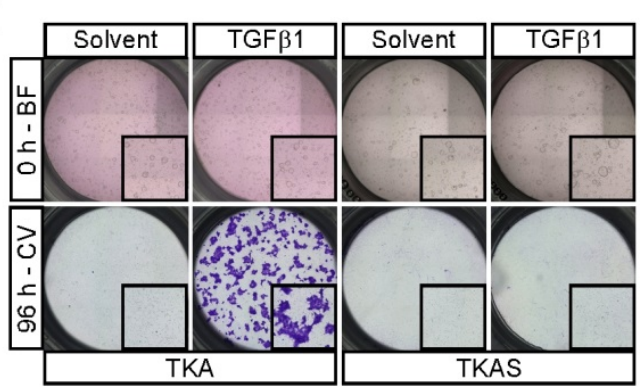

j

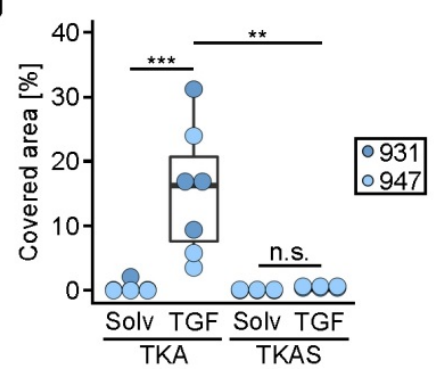

Fig. 5: The TGF $\beta 1$ response in TKA organoids is mediated by canonical TGF $\beta$ receptor/Smad signaling. a, Whole mount phase contrast microscopy of TKA organoids transduced with an empty vector (EV) or an expression vector for dominant negative TGFBR2 (TGFBR2DN), seeded in $3 \mathrm{mg} / \mathrm{ml}$ Matrigel, and treated with solvent or TGF $\beta 1$ for 72 h. Scale bar: $200 \mu \mathrm{m}$. b, Western blot expression analysis of the indicated proteins in control (EV) and TGFBR2DN-expressing TKA organoids (line 947) treated as in (a). Gsk3 $\beta$ detection served as loading control. Molecular weights of size standards are given in kDa. 
Panels ( $\mathbf{a}$ and $\mathbf{b}$ ) show representative results of two independent biological replicates for TKA organoid lines $931(n=2)$ and $947(n=2)$. c, Exemplary Boyden chamber invasion assays with control (EV) and TGFBR2DN-expressing TKA organoids (line 931) seeded in $3 \mathrm{mg} / \mathrm{ml}$ Matrigel. Bright field (BF) images were taken at $0 \mathrm{~h}$ of solvent and TGF $\beta 1$ treatment. Inserts: magnified views of the upper chambers. Invaded cells were visualized by crystal violet (CV) staining after $96 \mathrm{~h}$ of treatment. Inserts: magnified views of the bottom face of the Boyden chambers. d, Quantification of invasion experiments as shown in (c). Dots represent results of independent biological replicates (line 931: $n=3$; line 947: $n=4$ ). Dot color identifies the organoid lines. ${ }^{* * *} p<0.001$; Mann-Whitney $U$ test. e, Scheme of the Smad4 locus showing sgRNA target positions (red arrows) flanking exon 9 (black box) and the location of PCR primers used for genotyping. The distance between the sgRNA targets and the length of the PCR amplicon in Smad4 wt organoids are given in base pairs (bp). f, Results of genotyping PCRs with genomic DNA from Smad4 wt TKA and Smad4 mutant TKAS organoid lines as indicated. Sizes of DNA standards are given in kilo base pairs (kbp). g, Western blot expression analyses for Smad4 in TKA and TKAS organoids. Gsk3 $\beta$ detection served as loading control $(n=3)$. Molecular weights of size standards are given in $\mathrm{kDa}$. $\mathbf{h}$, Whole mount phase contrast microscopy of TKA and TKAS organoid lines seeded in $3 \mathrm{mg} / \mathrm{ml}$ Matrigel and treated with solvent or TGF $\beta 1$ for $72 \mathrm{~h}$. Inserts display a larger field of view at lower magnification. Scale bars: $200 \mu \mathrm{m}$. i, Boyden chamber invasion assays performed with TKA and TKAS organoids (line 931) as in (c). j, Quantification of invasion experiments as shown in (i) performed with TKA and TKAS organoid lines $931(n=4)$ and $947(n=3)$. Dots represent results of individual experiments. Dot color identifies the organoid lines. ${ }^{* * *} p=0.0006$, ${ }^{* *} p=0.0012$, n.s.: not significant $(p=0.39)$; Mann-Whitney $U$ test. For ( $\mathbf{d}$ and $\left.\mathbf{j}\right)$, exact $p$-values are provided in Supplementary table 1. 
a

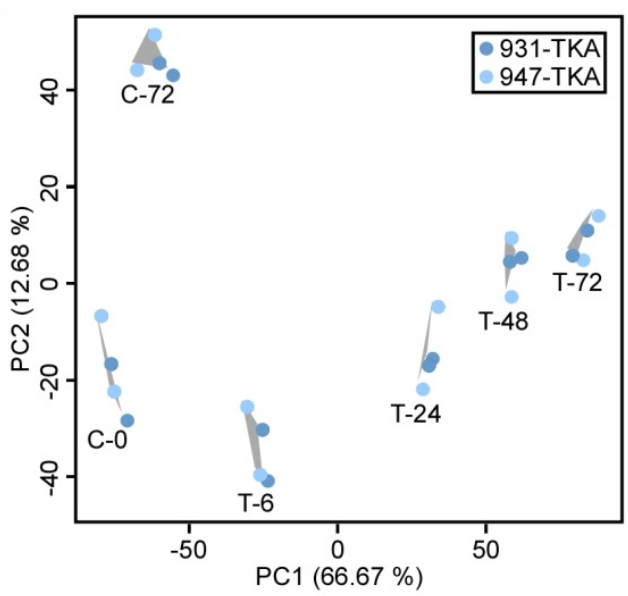

b

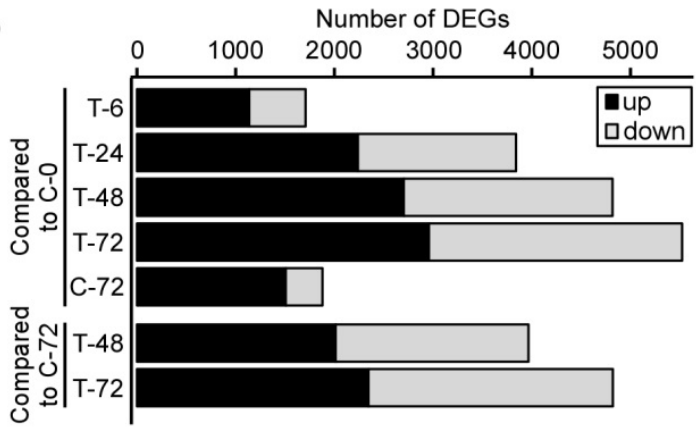

d

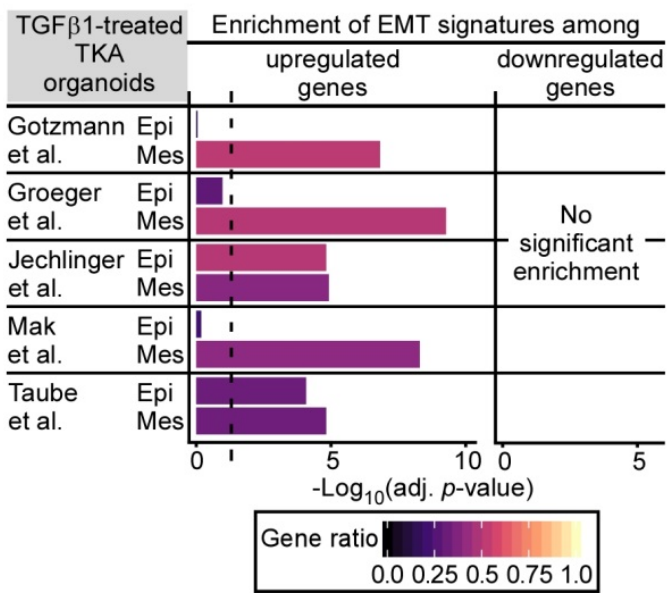

C

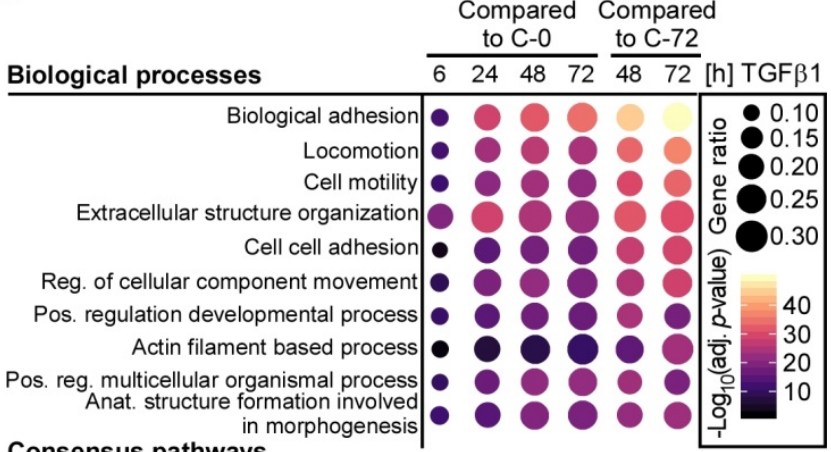

Consensus pathways

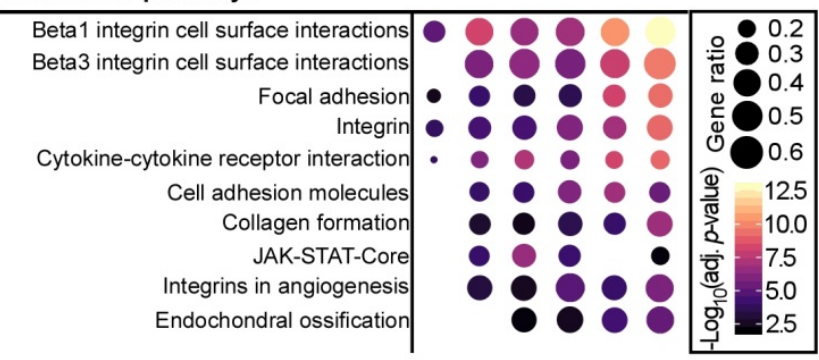

e

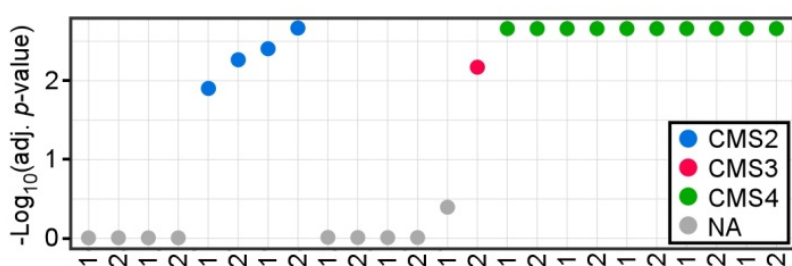

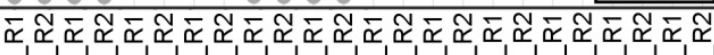

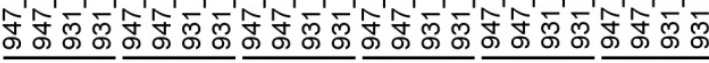

f

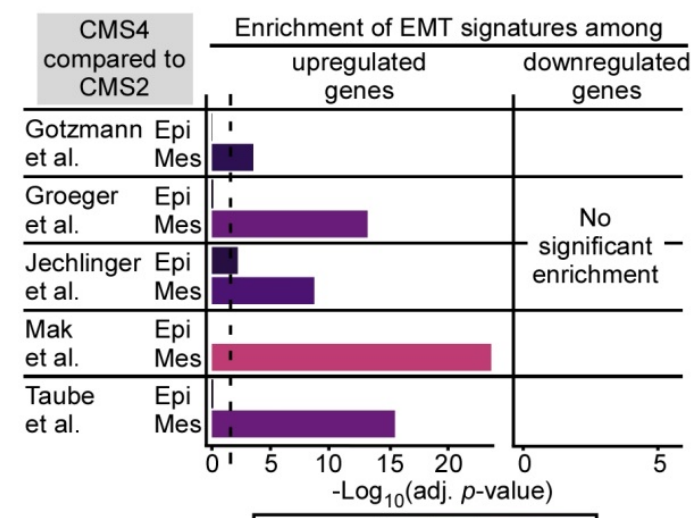

Gene ratio

Fig. 6: TGF $\beta 1$-induced global transcriptional deregulation features a partial EMT in TKA organoids. a, Principal component analysis (PCA) of RNA-seq data from TKA organoids seeded in $3 \mathrm{mg} / \mathrm{ml}$ Matrigel and treated with solvent (C) or TGF $\beta 1$ (T) as indicated. Results are based on two independent biological replicates for organoid lines $931(n=2)$ and $947(n=2)$. b, Numbers of differentially expressed genes (DEGs) were determined by 
performing pairwise comparisons of transcriptomes from TKA organoids harvested at the onset of the experiment ( $0 \mathrm{~h}$ of cultivation; C-0), cultivated for $72 \mathrm{~h}$ in solvent (C-72), and treated with TGF $\beta 1$ for the indicated periods of time. Black and grey segments of the bars: up- and downregulated genes, respectively. c, Functional enrichment analysis of genes upregulated upon TGF $\beta 1$ treatment (adjusted $p$-value $<0.01, \log _{2}(F C)>1$ ). The top ten $G O$ terms from the indicated categories are listed. Dot size: ratio of upregulated genes compared to all genes within a set. Dot color: $-\log _{10}(\operatorname{adjusted}$ [adj.] $p$-value) of the enrichments. d, Exclusive enrichment of mesenchymal components of published EMT signatures among genes significantly upregulated in TGF $\beta 1$-treated TKA organoids. The analyses were conducted for DEGs from TKA organoids treated with TGF $\beta 1$ for $72 \mathrm{~h}$ compared to cultivation for $72 \mathrm{~h}$ in solvent. Published EMT signatures were split into subsets comprising epithelial (Epi) and mesenchymal genes (Mes) and processed separately. The color encodes the gene ratio. Length of the bars depicts the $-\log _{10}$ of the adj. $p$-values. Dotted line: adj. $p$ value $=0.05$. e, The independent biological replicates $(R 1, R 2)$ of the transcriptomes as described in (a and $\mathbf{b}$ ) were assessed for resemblance to the four consensus molecular subtypes (CMS) of CRC. Colored dots and their positions depict the CMS type and the adj. $p$-value, respectively. NA: no significant classification possible. f, Functional enrichment analyses of EMT sub-signatures as described in (d) among genes differentially expressed in colon cancers classified as CMS4 and CMS2. The color encodes the gene ratio. Length of the bars depicts the $-\log _{10}$ of the adj. $p$-values. Dotted line: adj. $p$-value $=0.05$. 
a

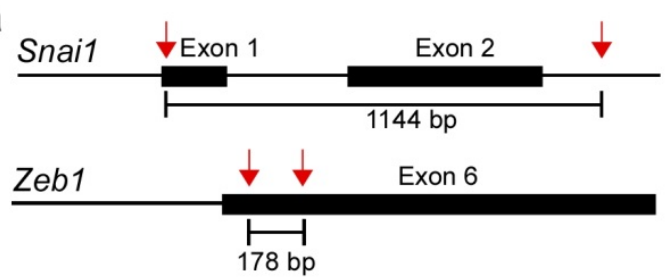

C
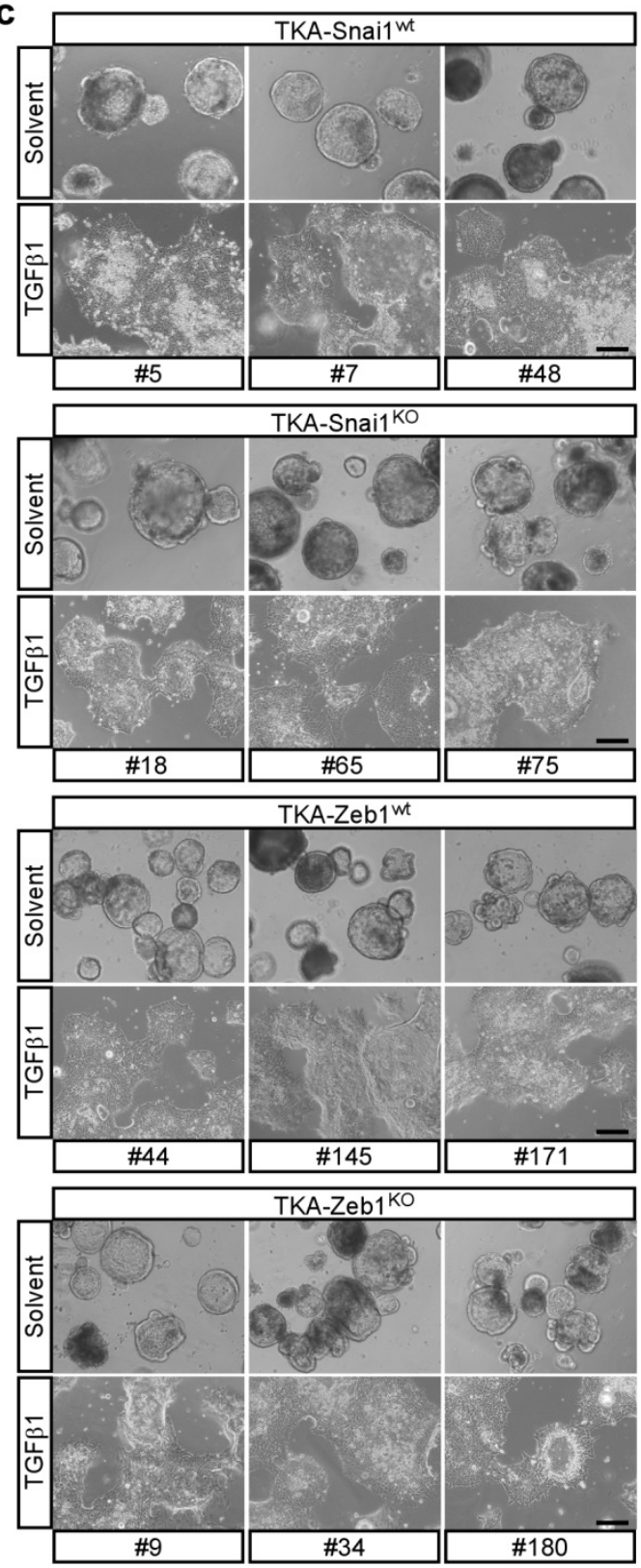

b

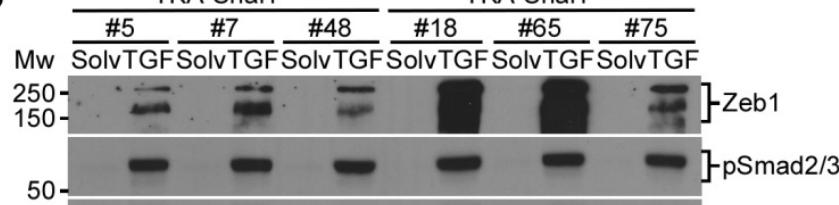

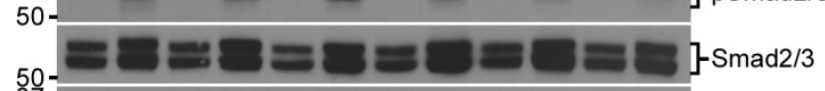

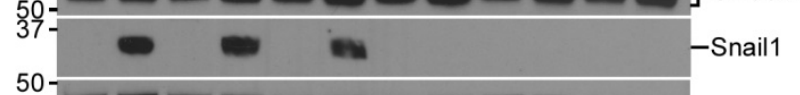

50 -Gsk3
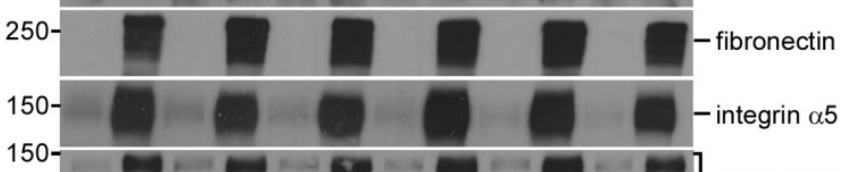

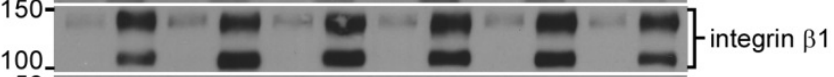

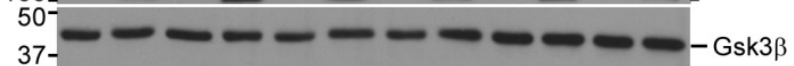

$150-2-6-6-\infty-6-6-6-6$ - Edherin

$100-$

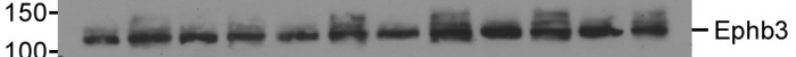

$50-$

$\begin{array}{llllllllllllll} & 1 & 2 & 3 & 4 & 5 & 6 & 7 & 8 & 9 & 10 & 11 & 12\end{array}$

$\frac{\text { TKA-Zeb1 } 1 \text { wt }}{\# 44} \# \frac{\text { TKA-Zeb1 } 1 \text { KO }}{\# 145} \# 171$

Mw SolvTGF SolvTGF $\frac{\# 1 \text { SolvTGF }}{\text { SolvTGF SolvTGF SolvTGF }}$
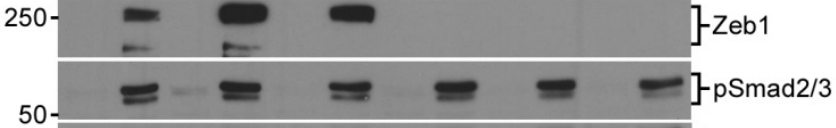

50 -

50
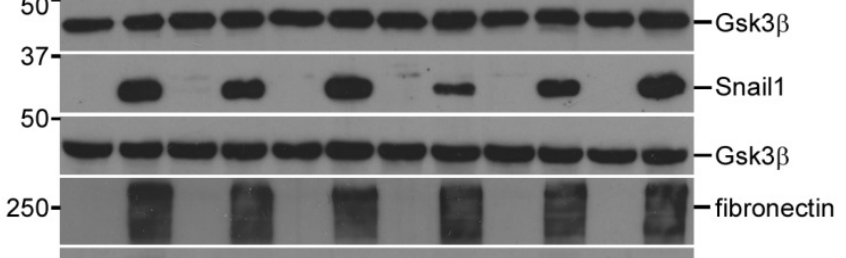

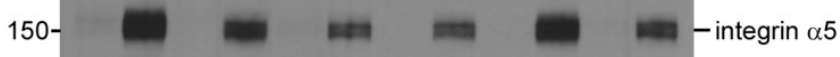

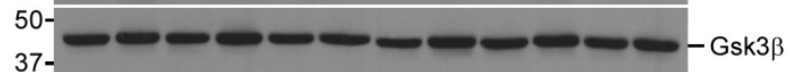

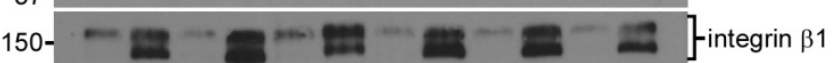

100

$150-----\infty----$ E-cadherin

150-

$108=$

$\begin{array}{rllllllllllll}17-G s k 3 \beta & \end{array}$

Fig. 7: TGFß1-induced collective invasion occurs independently from the EMT

transcription factors Snail1 and Zeb1. a, Schematics of the Snai1 and Zeb1 loci showing sgRNA target positions (red arrows) and relevant exons (black boxes). The size of the expected deletions is given in base pairs (bp). b. Western blot expression analyses of the proteins indicated in TKA-Snai $1^{\mathrm{wt}}$, TKA-Snai $1^{\mathrm{KO}}$, TKA-Zeb1 $1^{\mathrm{wt}}$, and TKA-Zeb1 ${ }^{\mathrm{KO}}$ organoids 
derived from line 815 seeded in $3 \mathrm{mg} / \mathrm{ml}$ Matrigel and treated with solvent (solv) or TGF $\beta 1$ (TGF) for 72 h. Gsk3 $\beta$ detection served as loading control $(n=3)$. Molecular weights of size standards are given in kDa. c, Morphological appearance of TKA-Snai $1^{\mathrm{wt}}(\# 5, \# 7, \# 48)$, TKASnai1 $^{\mathrm{KO}}(\# 18, \# 65, \# 75)$, TKA-Zeb1 $^{\mathrm{wt}}(\# 44, \# 145, \# 171)$, and TKA-Zeb1 ${ }^{\mathrm{KO}}(\# 9, \# 34, \# 180)$ organoids derived from line 815 cultured in Matrigel and treated with solvent or TGF $\beta 1$ for 72 h. Scale bars: $200 \mu \mathrm{m}$. 